\title{
A classification of generalized quantum statistics associated with basic classical Lie superalgebras
}

\author{
N.I. Stoilova ${ }^{\dagger}$ and J. Van der Jeugt \\ Department of Applied Mathematics and Computer Science, \\ University of Ghent, Krijgslaan 281-S9, B-9000 Gent, Belgium. \\ E-mails: Neli.Stoilova@UGent.be, Joris.VanderJeugt@UGent.be.
}

\begin{abstract}
Generalized quantum statistics such as para-statistics is usually characterized by certain triple relations. In the case of para-Fermi statistics these relations can be associated with the orthogonal Lie algebra $B_{n}=s o(2 n+1)$; in the case of paraBose statistics they are associated with the Lie superalgebra $B(0 \mid n)=o s p(1 \mid 2 n)$. In a previous paper, a mathematical definition of "a generalized quantum statistics associated with a classical Lie algebra $G$ " was given, and a complete classification was obtained. Here, we consider the definition of "a generalized quantum statistics associated with a basic classical Lie superalgebra $G$ ". Just as in the Lie algebra case, this definition is closely related to a certain $\mathbb{Z}$-grading of $G$. We give in this paper a complete classification of all generalized quantum statistics associated with the basic classical Lie superalgebras $A(m \mid n), B(m \mid n), C(n)$ and $D(m \mid n)$.
\end{abstract}

Running title: Classification of generalized statistics

PACS: 02.20.+b, 03.65.Fd, 05.30-d.

${ }^{\dagger}$ Permanent address: Institute for Nuclear Research and Nuclear Energy, Boul. Tsarigradsko Chaussee 72, 1784 Sofia, Bulgaria 


\section{Introduction}

A historically important extension of Bose and Fermi statistics has been known for 50 years, namely the para-Bose and para-Fermi statistics as developed by Green [1]. In para-statistics, the usual bilinear commutators or anti-commutators for bosons and fermions are replaced by certain trilinear or triple relations. For example, for $n$ pairs of para-Bose creation and annihilation operators $B_{i}^{\xi}(\xi= \pm$ and $i=1, \ldots, n)$, the defining relations are:

$$
\begin{aligned}
{\left[\left\{B_{j}^{\xi}, B_{k}^{\eta}\right\}, B_{l}^{\epsilon}\right] } & =(\epsilon-\xi) \delta_{j l} B_{k}^{\eta}+(\epsilon-\eta) \delta_{k l} B_{j}^{\xi} \\
\xi, \eta, \epsilon & = \pm \text { or } \pm 1 ; \quad j, k, l=1, \ldots, n
\end{aligned}
$$

Similar triple relations hold for the para-Fermi operators $F_{i}^{\xi}[1]$, see (1.1) in [2]. Both for para-Bose and para-Fermi statistics, there is a group theoretical setting. It was shown 3 . that the Lie algebra generated by the $2 n$ elements $F_{i}^{\xi}$, with $\xi= \pm$ and $i=1, \ldots, n$, subject to the para-Fermi relations is $B_{n}=s o(2 n+1)$ (as a Lie algebra defined by means of generators and relations).

Twenty years after the connection between para-Fermi statistics and the Lie algebra $s o(2 n+1)$, a new connection, between para-Bose statistics and the orthosymplectic Lie superalgebra $B(0 \mid n)=\operatorname{osp}(1 \mid 2 n)$ [4] was discovered [5]. The Lie superalgebra generated by $2 n$ odd elements $B_{i}^{\xi}$, with $\xi= \pm$ and $i=1, \ldots, n$, subject to the triple relations (1.1), is $\operatorname{osp}(1 \mid 2 n)$ (as a Lie superalgebra defined by means of generators and relations). Moreover, there is a certain representation of $\operatorname{ssp}(1 \mid 2 n)$, the so-called Bose representation $\mathcal{B}$, that yields the classical Bose relations, i.e. where the representatives $\mathcal{B}\left(B_{i}^{\xi}\right)$ satisfy the relations of classical Bose statistics. For more general para-Bose statistics, a class of infinite dimensional $\operatorname{osp}(1 \mid 2 n)$ representations needs to be investigated.

These examples show that para-statistics, as introduced by Green [1] and further developed by others (see [6] and the references therein), can be associated with representations of the Lie (super)algebras of class $B$ (namely $B_{n}$ and $B(0, n)$ ). Whether alternative types of generalized quantum statistics can be found in the framework of other classes of simple Lie algebras or superalgebras has been considered in particular by Palev [7]-[15]. Building upon his examples and inspired by the definition of creation and annihilation operators in [11, a mathematical definition of "generalized quantum statistics" was given in [2]. Furthermore, a complete classification was given of all the classes of generalized quantum statistics for the classical Lie algebras $A_{n}, B_{n}, C_{n}$ and $D_{n}$ [2], by means of their algebraic relations. In the present paper we make a similar classification for the basic classical Lie superalgebras.

For certain examples of quantum statistics associated with Lie superalgebras, see [14[15]. However, a complete classification was never made. A particular interesting example was described for the Lie superalgebra $\operatorname{sl}(1 \mid n)=A(0 \mid n-1)$ [15. For this superalgebra, a set of odd creation and annihilation operators was given [11], and it was shown that these $n$ pairs of operators $a_{i}^{\xi}$, with $\xi= \pm$ and $i=1, \ldots, n$, subject to the defining relations

$$
\begin{aligned}
& {\left[\left\{a_{i}^{+}, a_{j}^{-}\right\}, a_{k}^{+}\right]=\delta_{j k} a_{i}^{+}-\delta_{i j} a_{k}^{+},} \\
& {\left[\left\{a_{i}^{+}, a_{j}^{-}\right\}, a_{k}^{-}\right]=-\delta_{i k} a_{j}^{-}+\delta_{i j} a_{k}^{-},} \\
& \left\{a_{i}^{+}, a_{j}^{+}\right\}=\left\{a_{i}^{-}, a_{j}^{-}\right\}=0,
\end{aligned}
$$

$(i, j, k=1, \ldots, n)$, generate the special linear Lie superalgebra $s l(1 \mid n)$ (as a Lie superalgebra defined by means of generators and relations). Just as in the case of para-Bose relations, 
(1.2) has two interpretations. On the one hand, it describes the algebraic relations of a new kind of generalized statistics, in this case $A$-superstatistics or a statistics related to the Lie superalgebra $A(0 \mid n-1)$. On the other hand, (1.2) yields a set of defining relations for the Lie superalgebra $A(0 \mid n-1)$ in terms of generators and relations. Observe that certain microscopic and macroscopic properties of this statistics have already been studied [15.

A description similar to (1.2) for the Lie algebra $A_{n}$ was given for the first time by $\mathrm{N}$. Jacobson [16] in the context of "Lie triple systems". Therefore, this type of generators is often referred to as the "Jacobson generators". In this context, we shall mainly use the terminology "creation and annihilation operators (CAOs) for $\operatorname{sl}(1 \mid n)$ ".

Following the mathematical definition of "generalized quantum statistics associated with a Lie algebra", given in [2], this notion will be extended to Lie superalgebras $G$. This definition, and the corresponding classification method, are described in section III Just as for the case of Lie algebras, the method leads to a classification of certain gradings of $G$, and to regular subalgebras of $G$. In this process, Dynkin diagram techniques play a crucial role. For the basic classical Lie superalgebras however, the description by means of a Dynkin diagram is not unique: besides the so-called distinguished Dynkin diagram, other non-equivalent Dynkin diagrams exist [4], [17. This feature will make it harder to obtain a complete classification of all generalized quantum systems. In the remaining sections, the classification results are presented for all basic classical Lie superalgebras. A final section discusses some possible applications.

For the basic classical Lie superalgebras [4, we use the notation $A(m \mid n)=s l(m+1 \mid n+1)$, $B(m \mid n)=\operatorname{osp}(2 m+1 \mid 2 n), C(n)=\operatorname{osp}(2 \mid 2 n-2)$ and $D(m \mid n)=\operatorname{osp}(2 m \mid 2 n)$. The algebra $B(0 \mid n)=\operatorname{osp}(1 \mid 2 n)$ has a different structure and is usually considered separately (also here). For the classical simple Lie algebras, we use the notation $A_{n}=s l(n+1), B_{n}=s o(2 n+1)$, $C_{n}=s p(2 n)$ and $D_{n}=s o(2 n)$; note the difference between $C_{n}$ and $C(n)$. Note also that for trivial values of $m$ or $n$, a Lie superalgebra coincides with a Lie algebra: $\operatorname{sl}(r \mid 0)=\operatorname{sl}(0 \mid r)=$ $s l(r), B(m \mid 0)=B_{m}, D(m \mid 0)=D_{m}, D(0 \mid n)=C_{n}$.

\section{Definition and classification method}

Let $G$ be a basic classical Lie superalgebra. $G$ has a $\mathbb{Z}_{2}$-grading $G=G_{\overline{0}} \oplus G_{\overline{1}}$; an element $x$ of $G_{\overline{0}}$ is an even element $(\operatorname{deg}(x)=0)$, an element $y$ of $G_{\overline{1}}$ is an odd element $(\operatorname{deg}(y)=1)$. The elements which are purely even or odd are called homogeneous elements. The Lie superalgebra bracket is denoted by $\llbracket x, y \rrbracket$. In the universal enveloping algebra of $G$, this stands for

$$
\llbracket x, y \rrbracket=x y-(-1)^{\operatorname{deg}(x) \operatorname{deg}(y)} y x,
$$

if $x$ and $y$ are homogeneous. So the bracket can be a commutator or an anti-commutator.

Just as for a Lie algebra [2, a generalized quantum statistics associated with $G$ is determined by a set of $N$ creation operators $x_{i}^{+}$and $N$ annihilation operators $x_{i}^{-}$. Following the ideas of para-Bose statistics and those of [2], these $2 N$ operators should generate the Lie superalgebra $G$, subject to certain triple relations. Let $G_{+1}$ and $G_{-1}$ be the subspaces of $G$ spanned by these elements:

$$
G_{+1}=\operatorname{span}\left\{x_{i}^{+} ; i=1 \ldots, N\right\}, \quad G_{-1}=\operatorname{span}\left\{x_{i}^{-} ; i=1 \ldots, N\right\} .
$$


We do not require that these subspaces are homogeneous. The space $\llbracket G_{+1}, G_{+1} \rrbracket$ can be zero (in which case the creation operators mutually supercommute, as in (1.2)) or nonzero (as in (1.1)). A similar statement holds for the annihilation operators and $\llbracket G_{-1}, G_{-1} \rrbracket$. Putting $G_{ \pm 2}=\llbracket G_{ \pm 1}, G_{ \pm 1} \rrbracket$ and $G_{0}=\llbracket G_{+1}, G_{-1} \rrbracket$, the condition that $G$ is generated by the $2 N$ elements subject to triple relations only, leads 2 to the requirement that $G=$ $G_{-2} \oplus G_{-1} \oplus G_{0} \oplus G_{+1} \oplus G_{+2}$, and this must be a $\mathbb{Z}$-grading of $G$. Since these subspaces are not necessarily homogeneous, this $\mathbb{Z}$-grading is in general not consistent with the $\mathbb{Z}_{2}$-grading.

Just as in 2], we shall impose two further requirements: first of all, the generating elements $x_{i}^{ \pm}$must be root vectors of $G$. Secondly, $\omega\left(x_{i}^{+}\right)=x_{i}^{-}$, where $\omega$ is the standard antilinear anti-involutive mapping of $G$ (in terms of root vectors $e_{\alpha}, \omega$ satisfies $\omega\left(e_{\alpha}\right)=e_{-\alpha}$ ). This leads to the following definition, completely analogous as in [2]:

Definition 1 Let $G$ be a basic classical Lie superalgebra, with antilinear anti-involutive mapping $\omega$. A set of $2 N$ root vectors $x_{i}^{ \pm}(i=1, \ldots, N)$ is called a set of creation and annihilation operators for $G$ if:

- $\omega\left(x_{i}^{ \pm}\right)=x_{i}^{\mp}$,

- $G=G_{-2} \oplus G_{-1} \oplus G_{0} \oplus G_{+1} \oplus G_{+2}$ is a $\mathbb{Z}$-grading of $G$, with $G_{ \pm 1}=\operatorname{span}\left\{x_{i}^{ \pm}, i=\right.$ $1 \ldots, N\}$ and $G_{j+k}=\llbracket G_{j}, G_{k} \rrbracket$.

The algebraic relations $\mathcal{R}$ satisfied by the operators $x_{i}^{ \pm}$are the relations of a generalized quantum statistics (GQS) associated with $G$.

This is a mathematical generalization of quantum statistics. Whether all such GQS actually lead to physically acceptable quantum statistics remains to be seen; in this sense one should interpret our GQS as "candidates for generalizations of quantum statistics".

A GQS is characterized by a set $\left\{x_{i}^{ \pm}\right\}$of CAOs and the set of algebraic relations $\mathcal{R}$ they satisfy. A consequence of this definition is that $G$ is generated by $G_{-1}$ and $G_{+1}$, i.e. by the set of CAOs, and since $G_{j+k}=\llbracket G_{j}, G_{k} \rrbracket$, it follows that

$$
G=\operatorname{span}\left\{x_{i}^{\xi}, \llbracket x_{i}^{\xi}, x_{j}^{\eta} \rrbracket ; \quad i, j=1, \ldots, N, \xi, \eta= \pm\right\} .
$$

This implies that it is necessary and sufficient to give all relations of the following type:

(R1) The set of all linear relations between the elements $\llbracket x_{i}^{\xi}, x_{j}^{\eta} \rrbracket(\xi, \eta= \pm, i, j=1, \ldots, N)$.

(R2) The set of all triple relations of the form $\llbracket \llbracket x_{i}^{\xi}, x_{j}^{\eta} \rrbracket, x_{k}^{\zeta} \rrbracket=$ linear combination of $x_{l}^{\theta}$.

So $\mathcal{R}$ consists of a set of quadratic relations and a set of triple relations. Also, as a Lie superalgebra defined by generators and relations, $G$ is uniquely characterized by the set of generators $x_{i}^{ \pm}$subject to the relations $\mathcal{R}$.

A consequence of this definition is that $G_{0}$ itself is a subalgebra of $G$ spanned by root vectors of $G\left[2\right.$. It follows that $G_{0}$ is a regular subalgebra containing the Cartan subalgebra $H$ of $G$. By the adjoint action, the remaining $G_{i}$ 's are $G_{0}$-modules. Thus the technique of [2] can be used in order to obtain a complete classification of all GQS associated with $G$ :

1. Determine all regular subalgebras $G_{0}$ of $G$. If not yet contained in $G_{0}$, replace $G_{0}$ by $G_{0}+H$. 
2. For each regular subalgebra $G_{0}$, determine the decomposition of $G$ into simple $G_{0^{-}}$ modules $g_{k}(k=1,2, \ldots)$.

3. Investigate whether there exists a $\mathbb{Z}$-grading of $G$ of the form

$$
G=G_{-2} \oplus G_{-1} \oplus G_{0} \oplus G_{+1} \oplus G_{+2}
$$

where each $G_{i}$ is either directly a module $g_{k}$ or else a sum of such modules $g_{1} \oplus g_{2} \oplus \cdots$, such that $\omega\left(G_{+i}\right)=G_{-i}$.

If the $\mathbb{Z}$-grading is of the form (2.3) with $G_{ \pm 2} \neq 0$, we shall say that it has length 5 ; if $G_{+2}=0$ (then $G_{-2}=0$, but $G_{ \pm 1} \neq 0$ ), then the $\mathbb{Z}$-grading is of length 3 .

To find regular subalgebras one can use the method of (extended) Dynkin diagrams [18]. The second stage is straightforward by means of representation theoretical techniques. The third stage requires most of the work: one must try out all possible combinations of the $G_{0}$-modules $g_{k}$, and see whether it is possible to obtain a grading of the type (2.3). In this process, if one of the simple $G_{0}$-modules $g_{k}$ is such that $\omega\left(g_{k}\right)=g_{k}$, then it follows that this module should be part of $G_{0}$. In other words, such a case reduces essentially to another case with a larger regular subalgebra.

In the following sections we shall give a summary of the classification process for the basic classical Lie superalgebras $A(m \mid n), B(m \mid n), B(0 \mid n), D(m \mid n)$ and $C(n)$. Note that, in order to identify a GQS associated with $G$, it is sufficient to give only the set of CAOs, or alternatively, to give the subspace $G_{-1}$ (then the $x_{i}^{-}$are the root vectors of $G_{-1}$, and $\left.x_{i}^{+}=\omega\left(x_{i}^{-}\right)\right)$. The set $\mathcal{R}$ then consist of all quadratic relations (i.e. the linear relations between the elements $\left.\llbracket x_{i}^{\xi}, x_{j}^{\eta} \rrbracket\right)$ and all triple relations, and all of these relations follow from the known supercommutation relations in $G$. Because, in principle, $\mathcal{R}$ can be determined from the set $\left\{x_{i}^{ \pm} ; i=1, \ldots, N\right\}$, we will not always give it explicitly.

Finally, observe that two different sets of CAOs $\left\{x_{i}^{ \pm} ; i=1 \ldots, N\right\}$ and $\left\{y_{i}^{ \pm} ; i=1 \ldots, N\right\}$ (same $N$ ) are said to be isomorphic if, for a certain permutation $\tau$ of $\{1,2, \ldots, N\}$, the relations between the elements $x_{\tau(i)}^{ \pm}$and $y_{i}^{ \pm}$are the same. In that case, the regular subalgebra $G_{0}$ spanned by $\left\{\llbracket x_{i}^{+}, x_{j}^{-} \rrbracket\right\}$ is isomorphic (as a Lie superalgebra) to the regular subalgebra spanned by $\left\{\llbracket y_{i}^{+}, y_{j}^{-} \rrbracket\right\}$.

\section{The Lie superalgebra $A(m \mid n)$}

Let $G$ be the special linear Lie superalgebra $A(m \mid n) \equiv \operatorname{sl}(m+1 \mid n+1)$, consisting of traceless $(m+n+2) \times(m+n+2)$ matrices. The Cartan subalgebra $H$ of $G$ is the subspace of diagonal matrices. The root vectors of $G$ are known to be the elements $e_{j k}(j \neq k=1, \ldots, m+n+2)$, where $e_{j k}$ is a matrix with zeros everywhere except a 1 on the intersection of row $j$ and column $k$. The $\mathbb{Z}_{2}$-grading is such that $\operatorname{deg}\left(e_{j k}\right)=\theta_{j k}=\theta_{j}+\theta_{k}$, where

$$
\theta_{j}= \begin{cases}0 & \text { if } \quad j=1, \cdots, m+1 \\ 1 & \text { if } j=m+2, \cdots, m+n+2 .\end{cases}
$$

The root corresponding to $e_{j k}(j, k=1, \ldots, m+1)$ is given by $\epsilon_{j}-\epsilon_{k}$; for $e_{m+1+j, m+1+k}$ $(j, k=1, \ldots, n+1)$ it is $\delta_{j}-\delta_{k}$; and for $e_{j, m+1+k}$, resp. $e_{m+1+k, j}, \quad(j=1, \ldots, m+1$, $k=1, \ldots, n+1)$ it is $\epsilon_{j}-\delta_{k}$, resp. $\delta_{k}-\epsilon_{j}$. The anti-involution is such that $\omega\left(e_{j k}\right)=e_{k j}$. 
The distinguished set of simple roots and the distinguished Dynkin diagram of $A(m \mid n)$ are given in Table 1, and so is the extended distinguished Dynkin diagram.

To find regular subalgebras of $G=A(m \mid n)$, one should delete nodes from the Dynkin diagrams of $A(m \mid n)$ (first the ordinary, and then the extended). This goes in systematic steps. For each step, we shall investigate whether it leads to a grading of type (2.3).

Step 1. Delete node $i$ from the distinguished Dynkin diagram. Then the corresponding diagram is the Dynkin diagram of $G_{0}=s l(i) \oplus s l(m+1-i \mid n+1)$ for $i=1, \ldots, m+1$ and of $G_{0}=s l(m+1 \mid i-m-1) \oplus \operatorname{sl}(n+m+2-i)$ for $i=m+2, \ldots, m+n+1$. There are only two $G_{0}$-modules and

$$
\begin{aligned}
& G_{-1}=\operatorname{span}\left\{e_{k l} ; k=1, \ldots, i, l=i+1, \ldots, m+n+2\right\}, \\
& G_{+1}=\operatorname{span}\left\{e_{l k} ; k=1, \ldots, i, l=i+1, \ldots, m+n+2\right\} .
\end{aligned}
$$

Therefore $A(m \mid n)$ has a grading of length $3, A(m \mid n)=G_{-1} \oplus G_{0} \oplus G_{+1}$, and the number of creation and annihilation operators is $N=i(m+n+2-i)$.

The most interesting realizations are those with $i=1, i=m+n+1, i=2$ and $i=m+n$. We shall give the explicit supercommutation relations between the CAOs for some of these cases.

For $i=1, N=m+n+1$. Putting

$$
a_{j}^{-}=e_{1, j+1}, \quad a_{j}^{+}=e_{j+1,1}, \quad j=1, \cdots, m+n+1,
$$

the relations $\mathcal{R}$ are:

$$
\begin{aligned}
& \llbracket a_{j}^{+}, a_{k}^{+} \rrbracket=\llbracket a_{j}^{-}, a_{k}^{-} \rrbracket=0, \\
& \llbracket \llbracket a_{j}^{+}, a_{k}^{-} \rrbracket, a_{l}^{+} \rrbracket=(-1)^{\theta_{j+1}} \delta_{j k} a_{l}^{+}+\delta_{k l} a_{j}^{+}, \\
& \llbracket \llbracket a_{j}^{+}, a_{k}^{-} \rrbracket, a_{l}^{-} \rrbracket=-(-1)^{\theta_{j+1}} \delta_{j k} a_{l}^{-}-(-1)^{\theta_{j+1, k+1} \theta_{l+1}} \delta_{j l} a_{k}^{-} .
\end{aligned}
$$

For $m=0$, these are the relations of $A$-superstatistics [11, [15], see (1.2). Also for general $m$ and $n$, these relations have been considered in another context [14].

For $i=m+n+1, N=m+n+1$. Putting

$$
a_{j}^{-}=e_{j, m+n+2}, \quad a_{j}^{+}=e_{m+n+2, j}, \quad j=1, \cdots, m+n+1
$$

one finds:

$$
\begin{aligned}
& \llbracket a_{j}^{+}, a_{k}^{+} \rrbracket=\llbracket a_{j}^{-}, a_{k}^{-} \rrbracket=0, \\
& \llbracket \llbracket a_{j}^{+}, a_{k}^{-} \rrbracket, a_{l}^{+} \rrbracket=\delta_{j k} a_{l}^{+}-(-1)^{\theta_{k}} \delta_{k l} a_{j}^{+}, \\
& \llbracket \llbracket a_{j}^{+}, a_{k}^{-} \rrbracket, a_{l}^{-} \rrbracket=-\delta_{j k} a_{l}^{-}-(-1)^{\left(\theta_{j}+1\right)\left(\theta_{k}+1\right)} \delta_{j l} a_{k}^{-} .
\end{aligned}
$$

The relations (3.4) and (3.5) are similar; however the corresponding GQS are not isomorphic. For instance, in (3.4) there are $m$ even and $n+1$ odd pairs of CAOs, and in (3.5) there are $n$ even and $m+1$ odd pairs of CAOs.

For $i=2, N=2(m+n)$. One puts

$$
\begin{array}{lll}
a_{-, j}^{-}=e_{1, j+2}, & a_{+, j}^{-}=e_{2, j+2}, & j=1, \ldots, m+n, \\
a_{-, j}^{+}=e_{j+2,1}, & a_{+, j}^{+}=e_{j+2,2}, & j=1, \ldots, m+n .
\end{array}
$$


Then the corresponding relations $\operatorname{read}(\xi, \eta, \epsilon= \pm ; j, k, l=1, \ldots, m+n)$ :

$$
\begin{aligned}
& \llbracket a_{\xi j}^{+}, a_{\eta k}^{+} \rrbracket=\llbracket a_{\xi j}^{-}, a_{\eta k}^{-} \rrbracket=0, \\
& \llbracket a_{\xi j}^{+}, a_{-\xi k}^{-} \rrbracket=0, \quad j \neq k, \quad \\
& \llbracket a_{-j}^{+}, a_{-k}^{-} \rrbracket=\llbracket a_{+j}^{+}, a_{+k}^{-} \rrbracket, \quad j \neq k, \\
& \llbracket a_{+j}^{+}, a_{-j}^{-} \rrbracket=\llbracket a_{+k}^{+}, a_{-k}^{-} \rrbracket, \quad \text { for } \theta_{j}=\theta_{k}, \\
& \llbracket a_{-j}^{+}, a_{+j}^{-} \rrbracket=\llbracket a_{-k}^{+}, a_{+k}^{-} \rrbracket, \quad \text { for } \theta_{j}=\theta_{k}, \\
& \llbracket \llbracket a_{\xi j}^{+}, a_{\eta k}^{-} \rrbracket, a_{\epsilon l}^{+} \rrbracket=(-1)^{\operatorname{deg}\left(a_{\xi j}^{+}\right) \operatorname{deg}\left(a_{\eta k}^{-}\right)+\delta_{\xi,-\eta} \theta_{12} \operatorname{deg}\left(a_{\epsilon l}^{+}\right)} \delta_{\eta \epsilon} \delta_{j k} a_{\xi l}^{+}+\delta_{\xi \eta} \delta_{k l} a_{\epsilon j}^{+}, \\
& \llbracket \llbracket a_{\xi j}^{+}, a_{\eta k}^{-} \rrbracket, a_{\epsilon l}^{-} \rrbracket=-(-1)^{\operatorname{deg}\left(a_{\xi j}^{+}\right) \operatorname{deg}\left(a_{\eta k}^{-}\right)} \delta_{\xi \epsilon} \delta_{j k} a_{\eta l}^{-}-(-1)^{\theta_{j+2, k+2} \operatorname{deg}\left(a_{\epsilon l}^{-}\right)} \delta_{\xi \eta} \delta_{j l} a_{\epsilon k}^{-} .
\end{aligned}
$$

Such relations are definitely more complicated than (3.4) or (3.5). However, they are still proper defining relations for $A(m \mid n)$.

Step 2. Delete node $i$ and $j$ from the distinguished Dynkin diagram. We have $G_{0}=$ $H+\operatorname{sl}(i) \oplus \operatorname{sl}(j-i) \oplus \operatorname{sl}(m+1-j \mid n+1)$ for $1 \leq i<j \leq m+1, G_{0}=H+\operatorname{sl}(i) \oplus \operatorname{sl}(m+$ $1-i \mid j-m-1) \oplus \operatorname{sl}(m+n+2-j)$ for $1 \leq i \leq m+1, m+2 \leq j \leq m+n+1$ and $G_{0}=H+s l(m+1 \mid i-m-1) \oplus \operatorname{sl}(j-i) \oplus \operatorname{sl}(m+n+2-j)$ for $m+2 \leq i<j \leq m+n+1$. There are six simple $G_{0}$-modules. All the possible combinations of these modules give rise to gradings of length 5 . There are essentially three different ways in which these $G_{0}$-modules can be combined. To characterize these three cases, it is sufficient to give only $G_{-1}$ :

$$
\begin{aligned}
G_{-1}= & \operatorname{span}\left\{e_{k l}, e_{l p} ; k=1, \ldots, i, l=i+1, \ldots, j, p=j+1, \ldots, m+n+2\right\}, \\
& \operatorname{with} N=(j-i)(m+n+2-j+i) ; \\
G_{-1}= & \operatorname{span}\left\{e_{k l}, e_{p k} ; k=1, \ldots, i, l=i+1, \ldots, j, p=j+1, \ldots, m+n+2\right\}, \\
& \text { with } N=i(m+n+2-i) ; \\
G_{-1}= & \operatorname{span}\left\{e_{k l}, e_{l p} ; k=1, \ldots, i, p=i+1, \ldots, j, l=j+1, \ldots, m+n+2\right\}, \\
& \text { with } N=j(m+n+2-j) .
\end{aligned}
$$

Note that a part of the solutions in (3.8) and (3.9) are isomorphic to some of those given by (3.7). The isomorphic cases can be recognized as those having the same Dynkin diagram of $G_{0}$ and the same $N$-value.

For reasons explained earlier, we shall no longer give the corresponding set of relations explicitly for all possible cases. As an example, we consider here the case $j-i=1$ and (3.7). Then there are $N=m+n+1$ pairs of CAOs, which we can label as follows:

$$
\begin{aligned}
& a_{k}^{-}=e_{k, i+1}, \quad a_{k}^{+}=e_{i+1, k}, \quad k=1, \ldots, i \\
& a_{k}^{-}=e_{i+1, k+1}, \quad a_{k}^{+}=e_{k+1, i+1}, \quad k=i+1, \ldots, m+n+1 .
\end{aligned}
$$

Using

$$
\langle k\rangle=\left\{\begin{array}{lll}
0 & \text { if } \quad k=1, \ldots, i \\
1 & \text { if } \quad k=i+1, \ldots, m+n+1
\end{array}\right.
$$

the quadratic and triple relations now read:

$$
\begin{aligned}
& \llbracket a_{k}^{+}, a_{l}^{+} \rrbracket=\llbracket a_{k}^{-}, a_{l}^{-} \rrbracket=0, \quad k, l=1, \ldots, i \text { or } k, l=i+1, \ldots, m+n+1, \\
& \llbracket a_{k}^{-}, a_{l}^{+} \rrbracket=\llbracket a_{k}^{+}, a_{l}^{-} \rrbracket=0, \quad k=1, \ldots, i, l=i+1, \ldots, m+n+1,
\end{aligned}
$$




$$
\begin{aligned}
& \llbracket \llbracket a_{k}^{+}, a_{l}^{-} \rrbracket, a_{p}^{+} \rrbracket=(-1)^{\langle l\rangle+\langle p\rangle+\langle k\rangle \theta_{k+1, i+1}} \delta_{k l} a_{p}^{+}+(-1)^{\langle l\rangle+\langle p\rangle+(1-\langle l\rangle) \theta_{l, i+1}\left(\theta_{l k}+\theta_{k, i+1}\right)} \delta_{l p} a_{k}^{+}, \\
& k, l=1, \cdots, i, \text { or } k, l=i+1, \ldots, m+n+1, \\
& \llbracket \llbracket a_{k}^{+}, a_{l}^{-} \rrbracket, a_{p}^{-} \rrbracket=-(-1)^{\langle l\rangle+\langle p\rangle+\operatorname{deg}\left(a_{k}^{+}\right)\left[\langle k\rangle \theta_{k+1, l+1}+(1-\langle l\rangle) \theta_{l, i+1}\right]} \delta_{k p} a_{l}^{-} \\
& -(-1)^{\langle l\rangle+\langle p\rangle+\langle k\rangle \theta_{k+1, i+1}} \delta_{k l} a_{p}^{-}, \quad k, l=1, \cdots, i, \text { or } k, l=i+1, \ldots, m+n+1, \\
& \llbracket \llbracket a_{k}^{\xi}, a_{l}^{\xi} \rrbracket, a_{p}^{-\xi} \rrbracket=-(-1)^{\frac{1}{2} \theta_{p, i+1}\left[(1+\xi) \theta_{l+1, i+1}+(1-\xi) \theta_{k, l+1}\right]} \delta_{k p} a_{l}^{\xi} \\
& +(-1)^{\frac{1}{2}(1+\xi) \theta_{l+1, i+1}\left(\theta_{k, i+1}+\theta_{k, l+1}\right)} \delta_{l p} a_{k}^{\xi}, \quad k=1, \ldots, i, l=i+1, \ldots, m+n+1, \\
& \llbracket \llbracket a_{k}^{\xi}, a_{l}^{\xi} \rrbracket, a_{p}^{\xi} \rrbracket=0, \quad \xi= \pm ; k, l, p=1, \ldots, m+n+1 .
\end{aligned}
$$

Step 3. If we delete three or more nodes from the distinguished Dynkin diagram, the resulting $\mathbb{Z}$-gradings of $A(m \mid n)$ are no longer of the form (2.3). So these cases are not relevant for our classification.

Step 4. Next, we move on to the extended distinguished Dynkin diagram, also given in Table 1. If we delete node $i$ from this extended diagram, the remaining diagram is again (a non-distinguished Dynkin diagram) of type $A(m \mid n)$, so $G_{0}=G$, and there are no CAOs.

Step 5. If we delete node $i$ and $j(i<j)$ from the extended distinguished Dynkin diagram, then $A(m \mid n)=G_{-1} \oplus G_{0} \oplus G_{+1}$ with $G_{0}=H+s l(m \mid n+1)$ or $H+s l(m+1 \mid n)$ when the nodes are adjacent, and $G_{0}=H+\operatorname{sl}(k \mid l) \oplus s l(p \mid q)$ with $k+p=m+1$ and $l+q=n+1$ when the nodes are nonadjacent. Note that $p$ or $q$ can be zero: $s l(r \mid 0)=s l(0 \mid r)=s l(r)$. Now

$$
G_{-1}=\operatorname{span}\left\{e_{k l} ; k=i+1 \ldots, j, l \neq i+1, \ldots, j\right\} .
$$

The number of annihilation operators is $N=(j-i)(n+m+2-j+i)$. A part of these solutions are isomorphic to some of those of Step 1. The isomorphic cases are again characterized by the fact that their $G_{0}$ 's are isomorphic Lie superalgebras and their $N$-values coincide.

Step 6. If we delete nodes $i, j$ and $k$ from the extended distinguished Dynkin diagram $(i<j<k)$, then the corresponding $\mathbb{Z}$-gradings are of the form (2.3). If the three nodes are adjacent $G_{0}=H+s l(m-1 \mid n+1), H+s l(m \mid n)$ or $H+s l(m+1 \mid n-1)$. When two adjacent and one nonadjacent nodes are deleted, $G_{0}=H+s l(l \mid p) \oplus s l(q \mid r)$ with $l+q=m$, $p+r=n+1$ or $l+q=m+1, p+r=n$. If all three nodes are nonadjacent then $G_{0}=H+\operatorname{sl}(l \mid p) \oplus \operatorname{sl}(q \mid r) \oplus \operatorname{sl}(s \mid t)$ with $l+q+s=m+1, p+r+t=n+1$. One or two of these three Lie superalgebras is $s l(r \mid 0)=s l(0 \mid r)=s l(r)$. There are three different ways in which the corresponding $G_{0}$-modules can be combined. We give here only $G_{-1}$ :

$$
\begin{aligned}
G_{-1}= & \operatorname{span}\left\{e_{p s}, e_{s q} ; p=1, \ldots, i, k+1, \ldots, n+m+2, s=i+1, \ldots, j, q=j+1, \ldots, k\right\}, \\
& \text { with } N=(j-i)(n+m+2-j+i) ; \\
G_{-1}= & \operatorname{span}\left\{e_{p s}, e_{q p} ; p=1, \ldots, i, k+1, \ldots, n+m+2, s=i+1, \ldots, j, q=j+1, \ldots, k\right\}, \\
& \text { with } N=(k-i)(n+m+2+i-k) ; \\
G_{-1}= & \operatorname{span}\left\{e_{p q}, e_{q s} ; p=1, \ldots, i, k+1, \ldots, n+m+2, s=i+1, \ldots, j, q=j+1, \ldots, k\right\}, \\
& \text { with } N=(k-j)(n+m+2+j-k) .
\end{aligned}
$$

Again a part of these solutions are isomorphic to some of those in Step 2 (characterized by an isomorphic $G_{0}$ and the same $N$ ).

Step 7. If we delete four or more nodes from the extended distinguished Dynkin diagram, the corresponding $\mathbb{Z}$-grading of $A(m \mid n)$ has no longer the required properties (i.e. there are non-zero subspaces $G_{i}$ with $|i|>2$ ). 
Step 8. Next, one should repeat the process for all non-distinguished Dynkin diagrams of $G$ and their extensions. This is what makes the work harder than the corresponding classification for Lie algebras (which have only one Dynkin diagram and one extension). A general Dynkin diagram is determined by a general simple root system. All the systems of simple roots $\Pi_{S, T}$ of $A(m \mid n)$ are determined by two increasing sequences [4], 17]

$$
S=\left\{s_{1}<s_{2}<\ldots\right\} \text { and } T=\left\{t_{1}<t_{2}<\ldots\right\}
$$

and a sign:

$$
\Pi_{S, T}= \pm\left\{\epsilon_{1}-\epsilon_{2}, \epsilon_{2}-\epsilon_{3}, \ldots, \epsilon_{s_{1}}-\delta_{1}, \delta_{1}-\delta_{2}, \ldots, \delta_{t_{1}}-\epsilon_{s_{1}+1}, \ldots\right\}
$$

The Dynkin diagram itself looks like

where each dot can be a white or gray circle (depending upon whether the corresponding simple root is even or odd). Let $\pm\left(\eta_{i}-\eta_{j}^{\prime}\right)$ be the last element of $\Pi_{S, T}\left(\eta\right.$ and $\eta^{\prime}$ can be $\epsilon$ or $\delta)$. Then the zero node of the extended Dynkin diagram corresponds to $\pm\left(\eta_{j}^{\prime}-\epsilon_{1}\right)$ and it is uniquely determined.

If we repeat the whole procedure with the non-distinguished Dynkin diagrams (ordinary and extended), the only new result corresponds to Step 6 deleting three nonadjacent nodes from the extended Dynkin diagram. We have $G_{0}=H+\operatorname{sl}(l \mid p) \oplus s l(q \mid r) \oplus \operatorname{sl}(s \mid t)$ with $l+q+s=m+1, p+r+t=n+1$ and in some cases none of the three algebras is $s l(r \mid 0)=s l(0 \mid r)=s l(r)$. Just as in Step 2 or 6 , there are three different ways in which the $G_{0}$-modules can be combined; the explicit expression is left to the reader.

\section{The Lie superalgebras $B(m \mid n)$}

$G=B(m \mid n) \equiv \operatorname{osp}(2 m+1 \mid 2 n)$ is the subalgebra of $\operatorname{sl}(2 m+1 \mid 2 n)$ consisting of matrices of the form:

$$
\left(\begin{array}{ccccc}
a & b & u & x & x_{1} \\
c & -a^{t} & v & y & y_{1} \\
-v^{t} & -u^{t} & 0 & z & z_{1} \\
y_{1}^{t} & x_{1}^{t} & z_{1}^{t} & d & e \\
-y^{t} & -x^{t} & -z^{t} & f & -d^{t}
\end{array}\right)
$$

where $a$ is any $(m \times m)$-matrix, $b$ and $c$ are antisymmetric $(m \times m)$-matrices, $u$ and $v$ are $(m \times 1)$-matrices, $x, y, x_{1}, y_{1}$ are $(m \times n)$-matrices, $z$ and $z_{1}$ are $(1 \times n)$-matrices, $d$ is any $(n \times n)$-matrix, and $e$ and $f$ are symmetric $(n \times n)$-matrices. The even elements have $x=y=x_{1}=y_{1}=0, z=z_{1}=0$ and the odd elements are those with $a=b=c=0$, $u=v=0, d=e=f=0$. We shall consider $m=0$ separately in the next section. The Cartan subalgebra $H$ of $G$ is again the subspace of diagonal matrices $D$. Putting $\epsilon_{i}(D)=D_{i i}, i=1, \ldots, m$ and $\delta_{i}(D)=D_{2 m+i+1,2 m+i+1}, i=1, \ldots, n$, the even root vectors and corresponding roots of $G$ are given by:

$$
\begin{aligned}
e_{j k}-e_{k+m, j+m} & \leftrightarrow \epsilon_{j}-\epsilon_{k}, \quad j \neq k=1, \ldots, m, \\
e_{j, k+m}-e_{k, j+m} & \leftrightarrow \epsilon_{j}+\epsilon_{k}, \quad j<k=1, \ldots, m,
\end{aligned}
$$




$$
\begin{aligned}
e_{j+m, k}-e_{k+m, j} & \leftrightarrow-\epsilon_{j}-\epsilon_{k}, \quad j<k=1, \ldots, m, \\
e_{j, 2 m+1}-e_{2 m+1, j+m} & \leftrightarrow \epsilon_{j}, \quad j=1, \ldots, m, \\
e_{j+m, 2 m+1}-e_{2 m+1, j} & \leftrightarrow-\epsilon_{j}, \quad j=1, \ldots, m, \\
e_{2 m+1+j, 2 m+1+k}-e_{n+2 m+1+k, n+2 m+1+j} & \leftrightarrow \delta_{j}-\delta_{k}, \quad j \neq k=1, \ldots, n, \\
e_{2 m+1+j, 2 m+1+k+n}+e_{2 m+1+k, 2 m+1+j+n} & \leftrightarrow \delta_{j}+\delta_{k}, \quad j \leq k=1, \ldots, n, \\
e_{2 m+1+n+j, 2 m+1+k}+e_{2 m+1+n+k, 2 m+1+j} & \leftrightarrow-\delta_{j}-\delta_{k}, \quad j \leq k=1, \ldots, n,
\end{aligned}
$$

and the odd ones by

$$
\begin{aligned}
e_{j, 2 m+1+k}-e_{2 m+1+n+k, j+m} & \leftrightarrow \epsilon_{j}-\delta_{k}, \quad j=1, \ldots, m, k=1, \ldots, n, \\
e_{m+j, 2 m+1+k}-e_{2 m+1+n+k, j} & \leftrightarrow-\epsilon_{j}-\delta_{k}, \quad j=1, \ldots, m, k=1, \ldots, n, \\
e_{2 m+1,2 m+1+k}-e_{2 m+1+n+k, 2 m+1} & \leftrightarrow-\delta_{k}, \quad k=1, \ldots, n, \\
e_{j, 2 m+1+n+k}+e_{2 m+1+k, m+j} & \leftrightarrow \epsilon_{j}+\delta_{k}, \quad j=1, \ldots, m, k=1, \ldots, n, \\
e_{m+j, 2 m+1+n+k}+e_{2 m+1+k, j} & \leftrightarrow-\epsilon_{j}+\delta_{k}, \quad j=1, \ldots, m, k=1, \ldots, n, \\
e_{2 m+1,2 m+1+n+k}+e_{2 m+1+k, 2 m+1} & \leftrightarrow \delta_{k}, \quad k=1, \ldots, n .
\end{aligned}
$$

The distinguished set of simple roots and the corresponding Dynkin diagram of $B(m \mid n)$ are given in Table 1.

Step 1. Delete node $i$ from the distinguished Dynkin diagram. The corresponding diagram is the Dynkin diagram of $G_{0}=H+\operatorname{sl}(i) \oplus B(m \mid n-i)$ for $i=1, \ldots, n$ and of $G_{0}=$ $H+\operatorname{sl}(j \mid n) \oplus B_{m-j}$ for $i=n+j, j=1, \ldots, m$. There are four simple $G_{0}$-modules and $B(m \mid n)=G_{-2} \oplus G_{-1} \oplus G_{0} \oplus G_{+1} \oplus G_{+2}$, where for $i=1, \ldots n$ :

$$
\begin{aligned}
G_{-1}= & \operatorname{span}\left\{e_{2 m+1,2 m+1+n+k}+e_{2 m+1+k, 2 m+1}, e_{2 m+1+k, 2 m+1+n+l}+e_{2 m+1+l, 2 m+1+n+k},\right. \\
& e_{2 m+1+k, 2 m+1+l}-e_{n+2 m+1+l, n+2 m+1+k}, e_{p, 2 m+1+n+k}+e_{2 m+1+k, m+p}, \\
& \left.e_{m+p, 2 m+1+n+k}+e_{2 m+1+k, p} ; k=1, \ldots, i, l=i+1, \ldots, n, p=1, \ldots, m\right\} \\
& \text { with } N=2 i(m+n)-i(2 i-1)
\end{aligned}
$$

and for $i=n+j, j=1, \ldots m$ :

$$
\begin{aligned}
G_{-1}= & \operatorname{span}\left\{e_{2 m+1,2 m+1+n+k}+e_{2 m+1+k, 2 m+1}, e_{p, 2 m+1}-e_{2 m+1, m+p},\right. \\
& e_{p, m+q}-e_{q, m+p}, e_{p q}-e_{m+q, m+p}, e_{q, 2 m+1+n+k}+e_{2 m+1+k, m+q}, \\
& \left.e_{m+q, 2 m+1+n+k}+e_{2 m+1+k, q} ; k=1, \ldots, n, p=1, \ldots, j, q=j+1, \ldots, m\right\}, \\
& \text { with } N=2 i(m+n)-i(2 i-1) .
\end{aligned}
$$

It is interesting to give $\mathcal{R}$ for $i=n+m$, because then the number of creation or annihilation operators is $N=n+m$. One can label (and rescale) the CAOs as follows:

$$
\begin{aligned}
& b_{j}^{-} \equiv B_{j}^{-}=-\sqrt{2}\left(e_{2 m+1,2 m+1+n+j}+e_{2 m+1+j, 2 m+1}\right), \quad j=1, \ldots, n, \\
& b_{j}^{+} \equiv B_{j}^{+}=\sqrt{2}\left(e_{2 m+1,2 m+1+j}-e_{2 m+1+n+j, 2 m+1}\right), \quad j=1, \ldots, n, \\
& b_{n+j}^{-} \equiv F_{j}^{-}=\sqrt{2}\left(e_{j, 2 m+1}-e_{2 m+1, m+j}\right), \quad j=1, \ldots, m, \\
& b_{n+j}^{+} \equiv F_{j}^{+}=\sqrt{2}\left(e_{2 m+1, j}-e_{m+j, 2 m+1}\right), \quad j=1, \ldots, m .
\end{aligned}
$$


Note that

$$
\operatorname{deg}\left(b_{j}^{ \pm}\right)=\langle j\rangle=\left\{\begin{array}{lll}
1 & \text { if } & j=1, \ldots, n \\
0 & \text { if } j=n+1, \ldots, n+m
\end{array}\right.
$$

There are no quadratic relations, and $\mathcal{R}$ consists of triple relations only:

$$
\begin{gathered}
\llbracket \llbracket b_{j}^{\xi}, b_{k}^{\eta} \rrbracket, b_{l}^{\epsilon} \rrbracket=-2 \delta_{j l} \delta_{\epsilon,-\xi} \epsilon^{\langle l\rangle}(-1)^{\langle k\rangle\langle l\rangle} b_{k}^{\eta}+2 \epsilon^{\langle l\rangle} \delta_{k l} \delta_{\epsilon,-\eta} b_{j}^{\xi}, \\
\xi, \eta, \epsilon= \pm \text { or } \pm 1 ; \quad j, k, l=1, \ldots, n+m .
\end{gathered}
$$

Note that $B_{j}^{ \pm}, j=1, \ldots, n$ (resp. $\left.F_{k}^{ \pm}, k=1, \ldots, m\right)$ are para-Bose (resp. para-Fermi) CAOs, namely

$$
\begin{gathered}
{\left[\left\{B_{j}^{\xi}, B_{k}^{\eta}\right\}, B_{l}^{\epsilon}\right]=(\epsilon-\xi) \delta_{j l} B_{k}^{\eta}+(\epsilon-\eta) \delta_{k l} B_{j}^{\xi}} \\
\xi, \eta, \epsilon= \pm \text { or } \pm 1 ; \quad j, k, l=1, \ldots, n \\
{\left[\left[F_{j}^{\xi}, F_{k}^{\eta}\right], F_{l}^{\epsilon}\right]=\frac{1}{2}(\epsilon-\eta)^{2} \delta_{k l} F_{j}^{\xi}-\frac{1}{2}(\epsilon-\xi)^{2} \delta_{j l} F_{k}^{\eta}} \\
\xi, \eta, \epsilon= \pm \text { or } \pm 1 ; \quad j, k, l=1, \ldots, m
\end{gathered}
$$

The fact that $B(m \mid n)$ can be generated by $n$ pairs of para-Bose and $m$ pairs of para-Fermi operators has been discoved in [19].

Step 2. If we delete two or more nodes from the distinguished Dynkin diagram, the resulting $\mathbb{Z}$-gradings of $B(m \mid n)$ are no longer of the form (2.3).

Step 3. Now we continue with the extended Dynkin diagram, also given in Table 1. Delete node $i=0,1, \ldots, n+m$ from the extended distinguished Dynkin diagram. The remaining diagram is that of $G_{0}=B(m \mid n), A_{1} \oplus B(m \mid n-1), C_{2} \oplus B(m, n-2), \ldots, C_{n-1} \oplus B(m \mid 1)$, $C_{n} \oplus B_{m}, C(n+1) \oplus B_{m-1}, D(2 \mid n) \oplus B_{m-2}, \ldots, D(m-1 \mid n) \oplus A_{1}, D(m \mid n)$. In all these cases there is only one $G_{0}$-module, so there are no contributions to the classification.

Step 4. Delete the adjacent nodes $(i-1)$ and $i, i=2,3, \ldots, n$ from the extended distinguished Dynkin diagram. The remaining diagram is that of $\tilde{G}_{0}=A_{1} \oplus B(m \mid n-2)$ for $i=2$, of $\tilde{G}_{0}=C_{i-1} \oplus B(m \mid n-i)$ for $i=3, \ldots, n-1$ and of $\tilde{G}_{0}=C_{n-1} \oplus B_{m}$ for $i=n$. In each case there are seven $\tilde{G}_{0}$-modules $g_{k}$, one of which is invariant under $\omega$ (say $g_{1}$ ). Then one has to put $G_{0}=H+\tilde{G}_{0}+g_{1}$, and in each case one finds $G_{0} \equiv H+B(m \mid n-1)$. Now there are four $G_{0}$-modules and $B(m \mid n)=G_{-2} \oplus G_{-1} \oplus G_{0} \oplus G_{+1} \oplus G_{+2}$ with

$$
\begin{aligned}
G_{-1}= & \operatorname{span}\left\{e_{2 m+1,2 m+1+n+i}+e_{2 m+1+i, 2 m+1}, e_{2 m+1+i, 2 m+1+n+j}+e_{2 m+1+j, 2 m+1+n+i},\right. \\
& e_{2 m+1+i, 2 m+1+j}-e_{n+2 m+1+j, n+2 m+1+i}, i \neq j=1, \ldots, n \\
& \left.e_{k, 2 m+1+n+i}+e_{2 m+1+i, m+k}, e_{m+k, 2 m+1+n+i}+e_{2 m+1+i, k}, \quad k=1, \ldots, m\right\}
\end{aligned}
$$

and $N=2(m+n)-1$. Observe that in this case $G_{-2}=\operatorname{span}\left\{e_{2 m+1+i, 2 m+1+n+i}\right\}$, and all these cases are isomorphic to that of Step 1 with $i=1$.

Step 5. Delete the adjacent nodes $(i-1)$ and $i, i=n+j, j=1,2, \ldots, m$ from the extended distinguished Dynkin diagram. The remaining diagram is that of $\tilde{G}_{0}=C_{n} \oplus B_{m-1}$ for $j=1$, of $\tilde{G}_{0}=C(n+1) \oplus B_{m-2}$ for $j=2$, of $\tilde{G}_{0}=D(j-1 \mid n) \oplus B_{m-j}$ for $j=3, \ldots, m-2$, of $\tilde{G}_{0}=D(m-2 \mid n) \oplus A_{1}$ for $j=m-1$ and of $\tilde{G}_{0}=D(m-1 \mid n)$ for $j=m$. In each case there are five $\tilde{G}_{0}$-modules $g_{k}$, one of which is invariant under $\omega$ (say $g_{1}$ ). Then one has to 
put $G_{0}=H+\tilde{G}_{0}+g_{1}$, and in each case one finds $G_{0} \equiv H+B(m-1 \mid n)$. Now there are two $G_{0}$-modules and $B(m \mid n)=G_{-1} \oplus G_{0} \oplus G_{+1}$ with

$$
\begin{aligned}
G_{-1}= & \operatorname{span}\left\{e_{j, 2 m+1}-e_{2 m+1, j+m}, e_{j, k+m}-e_{k, j+m}, e_{j k}-e_{k+m, j+m}, j \neq k=1, \ldots, m ;\right. \\
& \left.e_{j, 2 m+1+n+l}+e_{2 m+1+l, m+j}, e_{j, 2 m+1+l}-e_{2 m+1+n+l, j+m}, l=1, \ldots, n\right\},
\end{aligned}
$$

and $N=2(m+n)-1$. All these cases are mutually isomorphic.

Step 6. Delete the nonadjacent nodes $i, j, i<j-1, j=3, \ldots, n$ from the extended distinguished Dynkin diagram. The remaining diagram is that of $\tilde{G}_{0}=C_{i} \oplus \operatorname{sl}(j-i) \oplus$ $B(m \mid n-j)$ (for $i=1$ instead of $C_{i}$ we have $A_{1}$ ). In each case there are seven $\tilde{G}_{0}$-modules $g_{k}$, one of which is invariant under $\omega$ (say $g_{1}$ ). Then one has to put $G_{0}=H+\tilde{G}_{0}+g_{1}$, and in each case one finds $G_{0} \equiv H+s l(j-i) \oplus B(m \mid n-j+i)$. Now there are four $G_{0}$-modules and $B(m \mid n)=G_{-2} \oplus G_{-1} \oplus G_{0} \oplus G_{+1} \oplus G_{+2}$. All these cases are isomorphic to those in Step 1 with $i=2, \ldots, n-1$.

Step 7. Delete the nonadjacent nodes $i, j, i=1, \ldots, n, j=n+1, \ldots, n+m[$ but $(i, j) \neq$ $(n, n+1)]$ from the extended distinguished Dynkin diagram. The remaining diagram is that of $\tilde{G}_{0}=C_{i} \oplus \operatorname{sl}(j-n \mid n-i) \oplus B_{n+m-j}$ (for $i=1$ instead of $C_{i}$ we have $A_{1}$ ). In each case there are seven $\tilde{G}_{0}$-modules $g_{k}$, one of which is invariant under $\omega$ (say $g_{1}$ ). Then one has to put $G_{0}=H+\tilde{G}_{0}+g_{1}$, and one always finds $G_{0} \equiv H+\operatorname{sl}(j-n \mid n-i) \oplus B(n+m-j \mid i)$. Now there are four $G_{0}$-modules and $B(m \mid n)=G_{-2} \oplus G_{-1} \oplus G_{0} \oplus G_{+1} \oplus G_{+2}$, where

$$
\begin{aligned}
G_{-1}= & \operatorname{span}\left\{e_{k, 2 m+1}-e_{2 m+1, k+m}, e_{2 m+1,2 m+1+n+l}+e_{2 m+1+l, 2 m+1},\right. \\
& e_{k, 2 m+1+n+p}+e_{2 m+1+p, m+k}, e_{k, 2 m+1+p}-e_{2 m+1+n+p, m+k}, \\
& e_{2 m+1+l, 2 m+1+n+p}+e_{2 m+1+p, 2 m+1+n+l}, e_{2 m+1+l, 2 m+1+p}-e_{2 m+1+n+p, 2 m+1+n+l}, \\
& e_{s, 2 m+1+n+l}+e_{2 m+1+l, m+s}, e_{m+s, 2 m+1+n+l}+e_{2 m+1+l, s}, \\
& k=1, \ldots, j-n, l=i+1, \ldots, n, p=1, \ldots, i, s=j+1-n, \ldots, m\},
\end{aligned}
$$

with $N=2(j-i)(m+n)-(j-i)(2(j-i)-1)$. All these cases are new (i.e. not isomorphic to an earlier case).

Step 8. Delete the nonadjacent nodes $i, j, i<j-1, i=n+1, \ldots, n+m-2, j=n+3, n+$ $4, \ldots, n+m$ from the extended distinguished Dynkin diagram. The remaining diagram is that of $\tilde{G}_{0}=D(i-n \mid n) \oplus \operatorname{sl}(j-i) \oplus B_{n+m-j}$ (for $i=n+1$ instead of $D(i-n \mid n)$ we have $C(n+1)$ ). In each case there are seven $\tilde{G}_{0}$-modules $g_{k}$, one of which is invariant under $\omega$ (say $\left.g_{1}\right)$. Then one has to put $G_{0}=H+\tilde{G}_{0}+g_{1}$, and one finds $G_{0} \equiv H+s l(j-i) \oplus B(m-j+i \mid n)$. Now there are four $G_{0}$-modules, and $B(m \mid n)=G_{-2} \oplus G_{-1} \oplus G_{0} \oplus G_{+1} \oplus G_{+2}$, where

$$
\begin{aligned}
G_{-1}= & \operatorname{span}\left\{e_{k, 2 m+1}-e_{2 m+1, k+m}, e_{k, m+l}-e_{l, m+k}, e_{k l}-e_{m+l, m+k},\right. \\
& e_{k, 2 m+1+n+p}+e_{2 m+1+p, m+k}, e_{k, 2 m+1+p}-e_{2 m+1+n+p, m+k}, \\
& k=i-n+1, i-n+2, \ldots, j-n, \\
& l=1,2, \ldots, i-n, j-n+1, j-n+2, \ldots, m, p=1,2, \ldots, n\},
\end{aligned}
$$

with $N=2(j-i)(m+n)-(j-i)(2(j-i)-1)$. All these cases are isomorphic to cases of Step 7.

Step 9. If we delete three or more nodes from the extended distinguished Dynkin diagram, the corresponding $\mathbb{Z}$-grading of $B(m \mid n)$ has no longer the required properties (i.e. there are non-zero subspaces $G_{i}$ with $|i|>2$ ). 
The next step consists of repeating this procedure for the non-distinguished Dynkin diagrams and their extensions. Following [17] one can obtain all such Dynkin diagrams of $B(m \mid n)$. We have repeated this procedure for all of them, leading to a lot of case studies but not leading to any new results (i.e. each case is isomorphic to one described already by means of the distinguished diagram).

\section{The Lie superalgebras $B(0 \mid n)$}

We consider the Lie superalgebra $B(0 \mid n)$ separately because the distinguished choice of the simple roots for $B(0 \mid n)$ is different than that of $B(m \mid n)$. In Table 1 the distinguished simple roots, the distinguished Dynkin diagram and the extended distinguished Dynkin diagram are given.

Step 1. Delete node $i, i=1, \ldots, n$ from the distinguished Dynkin diagram. The corresponding diagram is the Dynkin diagram of $G_{0}=H+\operatorname{sl}(i) \oplus B(0 \mid n-i)$. There are four simple $G_{0}$-modules and $B(0 \mid n)=G_{-2} \oplus G_{-1} \oplus G_{0} \oplus G_{+1} \oplus G_{+2}$, where

$$
\begin{aligned}
G_{-1}= & \operatorname{span}\left\{e_{1,1+n+k}+e_{1+k, 2 m+1}, e_{1+k, 1+n+l}+e_{1+l, 1+n+k}, e_{1+k, 1+l}-e_{n+1+l, n+1+k},\right. \\
& k=1, \ldots, i, l=i+1, \ldots, n,\}
\end{aligned}
$$

with $N=2 i(n-i)+i$. We give $\mathcal{R}$ for $i=n$, because then the number of creation or annihilation operators is $N=n$. One can label the CAOs as follows:

$$
\begin{aligned}
& B_{j}^{-}=-\sqrt{2}\left(e_{1,1+n+j}+e_{1+j, 1}\right), \quad j=1, \ldots, n, \\
& B_{j}^{+}=\sqrt{2}\left(e_{1,1+j}-e_{1+n+j, 1}\right), \quad j=1, \ldots, n .
\end{aligned}
$$

These are all odd generators of $B(0 \mid n)$ and the relations $\mathcal{R}$ consists of the triple para-Bose relations given already in (4.8).

Step 2. If we delete two or more nodes from the distinguished Dynkin diagram, the resulting $\mathbb{Z}$-gradings of $B(0 \mid n)$ are no longer of the form (2.3).

Step 3. Delete node $i, i=0,1, \ldots, n$ from the extended distinguished Dynkin diagram. The remaining diagram is that of $G_{0}=B(0 \mid n), A_{1} \oplus B(0 \mid n-1), C_{2} \oplus B(0, n-2), \ldots, C_{n-1} \oplus B(0 \mid 1)$, $C_{n}$. In all these cases there is only one $G_{0}$-module, so there are no contributions to the classification.

Step 4. Delete the adjacent nodes $(i-1)$ and $i, i=2,3, \ldots, n$ from the extended distinguished Dynkin diagram. The remaining diagram is that of $\tilde{G}_{0}=A_{1} \oplus B(0 \mid n-2)$ for $i=2$, of $\tilde{G}_{0}=C_{i-1} \oplus B(0 \mid n-i)$ for $i=3, \ldots, n-1$ and of $\tilde{G}_{0}=C_{n-1}$ for $i=n$. In each case there are seven $\tilde{G}_{0}$-modules $g_{k}$, one of which is invariant under $\omega$ (say $g_{1}$ ). Then one has to put $G_{0}=H+\tilde{G}_{0}+g_{1}$, and in each case one finds $G_{0} \equiv H+B(0 \mid n-1)$. Now there are four $G_{0}$-modules and $B(0 \mid n)=G_{-2} \oplus G_{-1} \oplus G_{0} \oplus G_{+1} \oplus G_{+2}$ with

$$
\begin{aligned}
G_{-1}= & \operatorname{span}\left\{e_{1,1+n+i}+e_{1+i, 1}, e_{1+i, 1+n+j}+e_{1+j, 1+n+i},\right. \\
& \left.e_{1+i, 1+j}-e_{n+1+j, n+1+i}, i \neq j=1, \ldots, n\right\},
\end{aligned}
$$

$N=2 n-1$, and $G_{-2}=\operatorname{span}\left\{e_{1+i, 1+n+i}\right\}$. All these cases are isomorphic to those of Step 1 with $i=1$. 
Step 5. Delete the nonadjacent nodes $i, j$ from the extended distinguished Dynkin diagram. The remaining diagram is that of $\tilde{G}_{0}=C_{i} \oplus s l(j-i) \oplus B(0 \mid n-j)$ (for $i=1$, $\tilde{G}_{0}=$ $A_{1} \oplus \operatorname{sl}(j-1) \oplus B(0 \mid n-j)$; for $\left.j=n, \tilde{G}_{0}=C_{i} \oplus \operatorname{sl}(n-i)\right)$. In each case there are seven $\tilde{G}_{0^{-}}$ modules $g_{k}$, one of which is invariant under $\omega$ (say $g_{1}$ ). Then one has to put $G_{0}=H+\tilde{G}_{0}+g_{1}$, and in each case one finds $G_{0} \equiv H+s l(j-i) \oplus B(0 \mid n-j+i)$. Now there are four $G_{0}$-modules and $B(0 \mid n)=G_{-2} \oplus G_{-1} \oplus G_{0} \oplus G_{+1} \oplus G_{+2}$. All these cases are isomorphic to those in Step 1 with $i=2, \ldots, n-1$.

Step 6. If we delete three or more nodes from the extended distinguished Dynkin diagram, the corresponding $\mathbb{Z}$-grading of $B(0 \mid n)$ has no longer the required properties (i.e. there are non-zero subspaces $G_{i}$ with $|i|>2$ ).

In the case of $B(0 \mid n)$ any other choice of simple roots is equivalent to the distinguished choice, so there are no more cases to study.

\section{The Lie superalgebras $D(m \mid n)$}

$G=D(m \mid n) \equiv \operatorname{osp}(2 m \mid 2 n)$ is the subalgebra of $\operatorname{sl}(2 m \mid 2 n)$ consisting of matrices of the form (4.1) with the middle row and column deleted. The Cartan subalgebra $H$ of $G$ is again the subspace of diagonal matrices $D$. Putting $\epsilon_{i}(D)=d_{i i}, i=1, \ldots, m, \delta_{i}(D)=d_{2 m+i, 2 m+i}$, $i=1, \ldots, n$, the even root vectors and corresponding roots of $G$ are given by:

$$
\begin{array}{rlrl}
e_{j k}-e_{k+m, j+m} & \leftrightarrow \epsilon_{j}-\epsilon_{k}, \quad j \neq k=1, \ldots, m, \\
e_{j, k+m}-e_{k, j+m} & \leftrightarrow \epsilon_{j}+\epsilon_{k}, \quad j<k=1, \ldots, m, \\
e_{j+m, k}-e_{k+m, j} & \leftrightarrow-\epsilon_{j}-\epsilon_{k}, \quad j<k=1, \ldots, m, \\
e_{2 m+j, 2 m+k}-e_{n+2 m+k, n+2 m+j} & \leftrightarrow \delta_{j}-\delta_{k}, \quad j \neq k=1, \ldots, n, \\
e_{2 m+j, 2 m+k+n}+e_{2 m+k, 2 m+j+n} & \leftrightarrow \delta_{j}+\delta_{k}, & j \leq k=1, \ldots, n, \\
e_{2 m+n+j, 2 m+k}+e_{2 m+n+k, 2 m+j} & \leftrightarrow-\delta_{j}-\delta_{k}, & j \leq k=1, \ldots, n,
\end{array}
$$

and the odd root vectors and roots by:

$$
\begin{aligned}
e_{j, 2 m+k}-e_{2 m+n+k, j+m} \leftrightarrow \epsilon_{j}-\delta_{k}, & j=1, \ldots, m, k=1, \ldots, n, \\
e_{m+j, 2 m+k}-e_{2 m+n+k, j} \leftrightarrow-\epsilon_{j}-\delta_{k}, & j=1, \ldots, m, k=1, \ldots, n, \\
e_{j, 2 m+n+k}+e_{2 m+k, m+j} \leftrightarrow \epsilon_{j}+\delta_{k}, & j=1, \ldots, m, k=1, \ldots, n, \\
e_{m+j, 2 m+n+k}+e_{2 m+k, j} \leftrightarrow-\epsilon_{j}+\delta_{k}, & j=1, \ldots, m, k=1, \ldots, n .
\end{aligned}
$$

The distinguished set of simple roots, the corresponding Dynkin diagram and its extension are given in Table 1.

Step 1. Delete node $i, i=1, \ldots, m+n-2$ from the distinguished Dynkin diagram. The corresponding diagram is that of $G_{0}=H+D(m \mid n-1)$ for $i=1$, of $G_{0}=H+\operatorname{sl}(i) \oplus D(m \mid n-i)$ for $i=2, \ldots, n-1$, of $G_{0}=H+\operatorname{sl}(n) \oplus D_{m}$ for $i=n$, of $G_{0}=H+s l(i-n \mid n) \oplus D_{m+n-i}$ for $i=n+1, \ldots, m+n-3$ and of $G_{0}=H+\operatorname{sl}(m-2 \mid n) \oplus A_{1} \oplus A_{1}$ for $i=m+n-2$. There are four simple $G_{0}$-modules and $D(m \mid n)=G_{-2} \oplus G_{-1} \oplus G_{0} \oplus G_{+1} \oplus G_{+2}$, where for, $i=1, \ldots n$ :

$$
G_{-1}=\operatorname{span}\left\{e_{2 m+j, 2 m+n+k}+e_{2 m+k, 2 m+j+n}, e_{2 m+j, 2 m+k}-e_{n+2 m+k, n+2 m+j},\right.
$$




$$
\begin{aligned}
& e_{l, 2 m+n+j}+e_{2 m+j, m+l}, e_{m+l, 2 m+n+j}+e_{2 m+j, l} \\
& j=1, \ldots, i, k=i+1, \ldots, n, l=1, \ldots, m\}
\end{aligned}
$$

with $N=2 i(m+n-i)$; whereas for $i=n+1, \ldots m+n-2$ :

$$
\begin{aligned}
G_{-1}= & \operatorname{span}\left\{e_{k, 2 m+n+j}+e_{2 m+j, m+k}, e_{m+k, 2 m+n+j}+e_{2 m+j, k},\right. \\
& e_{l, m+k}-e_{k, m+l}, e_{l k}-e_{m+k, m+l} ; \\
& j=1, \ldots, n, k=i-n+1, \ldots, m, l=1, \ldots, i-n,\},
\end{aligned}
$$

with $N=2 i(m+n-i)$.

Step 2. Delete node $m+n-1$ or $m+n$ from the distinguished Dynkin diagram. The corresponding diagram is the Dynkin diagram of $G_{0}=H+\operatorname{sl}(m \mid n)$. There are two simple $G_{0}$-modules and $D(m \mid n)=G_{-1} \oplus G_{0} \oplus G_{+1}$, where, for $m+n-1$ :

$$
\begin{aligned}
G_{-1}= & \operatorname{span}\left\{e_{j, m+k}-e_{k, m+j}, j<k=1, \ldots, m-1,\right. \\
& e_{2 m+q, 2 m+n+s}+e_{2 m+s, 2 m+n+q}, q \leq s=1, \ldots, n, \\
& e_{l m}+e_{2 m, m+l}, e_{l, 2 m+n+p}+e_{2 m+p, m+l}, e_{2 m, 2 m+n+p}+e_{2 m+p, m}, \\
& l=1, \ldots, m-1, p=1, \ldots, n\}
\end{aligned}
$$

and for $m+n$ :

$$
\begin{aligned}
G_{-1}= & \operatorname{span}\left\{e_{j, m+k}-e_{k, m+j}, j<k=1, \ldots, m,\right. \\
& e_{2 m+p, 2 m+n+s}+e_{2 m+s, 2 m+n+p}, p \leq s=1, \ldots, n, \\
& \left.e_{j, 2 m+n+l}+e_{2 m+l, m+j}, j=1, \ldots, m, l=1, \ldots, n\right\} .
\end{aligned}
$$

Both cases have $N=(m+n)(m+n+1) / 2-m$, and they are isomorphic.

Step 3. Upon deleting two nodes $i$ and $j$ (except $i=m+n-1, j=m+n$ ) or more from the distinguished Dynkin diagram of $D(m \mid n)$, the corresponding $\mathbb{Z}$-gradings have no longer the required property (there are non-zero $G_{i}$ with $|i|>2$ ).

Step 4. Delete node $m+n-1$ and $m+n$ from the distinguished Dynkin diagram. We have $G_{0}=H+\operatorname{sl}(m-1 \mid n)$. There are six simple $G_{0}$-modules. All the possible combinations of these modules give rise to gradings of the form (2.3). There are essentially three different ways in which these $G_{0}$-modules can be combined. To characterize these three cases, it is sufficient to give only $G_{-1}$ :

$$
\begin{aligned}
G_{-1}= & \operatorname{span}\left\{e_{j m}-e_{2 m, m+j}, e_{j, 2 m}-e_{m, m+j}, e_{m, 2 m+k}-e_{2 m+n+k, 2 m}, e_{m, 2 m+n+k}+e_{2 m+k, 2 m} ;\right. \\
& j=1, \ldots, m-1, k=1, \ldots, n\},
\end{aligned}
$$

with $N=2(m+n-1)$;

$$
\begin{aligned}
G_{-1}= & \operatorname{span}\left\{e_{j m}-e_{2 m, m+j}, e_{2 m, 2 m+n+k}+e_{2 m+k, m}, e_{m+j, l}-e_{m+l, j},\right. \\
& e_{m+j, 2 m+k}-e_{2 m+n+k, j}, e_{2 m+n+k, 2 m+p}+e_{2 m+n+p, 2 m+k} ; j=1, \ldots, m-1, \\
& k=1, \ldots, n, j<l=1, \ldots, m-1, k \leq p=1, \ldots, n\},
\end{aligned}
$$

with $N=(m+n)(m+n+1) / 2-m$;

$$
\begin{aligned}
G_{-1}= & \operatorname{span}\left\{e_{j, m+l}-e_{l, m+j}, e_{j, 2 m+n+k}+e_{2 m+k, m+j}, e_{2 m+k, 2 m+n+p}+e_{2 m+p, 2 m+n+k},\right. \\
& e_{m+j, m}-e_{2 m, j}, e_{2 m, 2 m+k}-e_{2 m+n+k, m} ; j=1, \ldots, m-1, \\
& k=1, \ldots, n, j<l=1, \ldots, m-1, k \leq p=1, \ldots, n\},
\end{aligned}
$$


with $N=(m+n)(m+n+1) / 2-m$. The cases (6.6) and (6.7) are isomorphic.

Step 5. Delete node $i, i=0,1, \ldots, n+m$ from the extended distinguished Dynkin diagram. The remaining diagram is that of $G_{0}=D(m \mid n), A_{1} \oplus D(m \mid n-1), C_{2} \oplus D(m \mid n-2), \ldots$, $C_{n-1} \oplus D(m \mid 1), C_{n} \oplus D_{m}, C(n+1) \oplus D_{m-1}, D(2 \mid n) \oplus D_{m-2}, \ldots, D(m-2 \mid n) \oplus A_{1} \oplus A_{1}, D(m \mid n)$, $D(m \mid n)$. In all these cases there is only one $G_{0}$-module, so there are no contributions to the classification.

Step 6. Delete the adjacent nodes $(i-1)$ and $i, i=2,3, \ldots, n$ from the extended distinguished Dynkin diagram. The remaining diagram is that of $\tilde{G}_{0}=A_{1} \oplus D(m \mid n-2)$ for $i=2$, of $\tilde{G}_{0}=C_{i-1} \oplus D(m \mid n-i)$ for $i=3, \ldots, n-1$ and of $\tilde{G}_{0}=C_{n-1} \oplus D_{m}$ for $i=n$. In each case there are seven $\tilde{G}_{0}$-modules $g_{k}$, one of which is invariant under $\omega$ (say $g_{1}$ ). Then one has to put $G_{0}=H+\tilde{G}_{0}+g_{1}$, and in each case one finds $G_{0} \equiv H+D(m \mid n-1)$. Now there are four $G_{0}$-modules and $D(m \mid n)=G_{-2} \oplus G_{-1} \oplus G_{0} \oplus G_{+1} \oplus G_{+2}$ with

$$
\begin{aligned}
G_{-1}= & \operatorname{span}\left\{e_{2 m+k, 2 m+n+i}+e_{2 m+i, 2 m+n+k}, e_{2 m+i, 2 m+k}-e_{2 m+n+k, 2 m+n+i}, i \neq k=1, \ldots, n,\right. \\
& \left.e_{l, 2 m+n+i}+e_{2 m+i, m+l}, e_{m+l, 2 m+n+i}+e_{2 m+i, l}, \quad l=1, \ldots, m\right\}
\end{aligned}
$$

$N=2(m+n-1)$ and $G_{-2}=\operatorname{span}\left\{e_{2 m+i, 2 m+n+i}\right\}$. All these cases are isomorphic to that of Step 1 with $i=1$.

Step 7. Delete the adjacent nodes $(i-1)$ and $i, i=n+1, \ldots, m+n-1$ from the extended distinguished Dynkin diagram. The remaining diagram is that of $\tilde{G}_{0}=C_{n} \oplus D_{m-1}$ for $i=n+1$, of $\tilde{G}_{0}=C(n+1) \oplus D_{m-2}$ for $i=n+2$, of $\tilde{G}_{0}=D(i-n-1 \mid n) \oplus D_{m+n-i}$ for $i=n+3, \ldots, m+n-3$, of $\tilde{G}_{0}=D(m-3 \mid n) \oplus A_{1} \oplus A_{1}$ for $i=m+n-2$ and of $\tilde{G}_{0}=D(m-2 \mid n) \oplus A_{1}$ for $i=m+n-1$. In each case there are five $\tilde{G}_{0}$-modules $g_{k}$, one of which is invariant under $\omega$ ( say $g_{1}$ ). Then one has to put $G_{0}=H+\tilde{G}_{0}+g_{1}$, and in each case one finds $G_{0} \equiv H+D(m-1 \mid n)$. Now there are two $G_{0}$-modules and $D(m \mid n)=G_{-1} \oplus G_{0} \oplus G_{+1}$ with

$$
\begin{aligned}
G_{-1}= & \operatorname{span}\left\{e_{i-n, 2 m+n+j}+e_{2 m+j, m+i-n}, e_{i-n, 2 m+j}-e_{2 m+n+j, m+i-n}, j=1, \ldots, n,\right. \\
& \left.e_{k, m+i-n}-e_{i-n, m+k}, e_{i-n, k}-e_{m+k, m+i-n}, i-n \neq k=1, \ldots, m\right\}
\end{aligned}
$$

and $N=2(m+n-1)$. All these cases are mutually isomorphic.

Step 8. Delete the adjacent nodes $m+n-1$ and $m+n$ from the extended distinguished Dynkin diagram. The remaining diagram is that of $G_{0} \equiv D(m-1 \mid n)$. There are two $G_{0}$-modules and $D(m \mid n)=G_{-1} \oplus G_{0} \oplus G_{+1}$ with

$$
\begin{aligned}
G_{-1}= & \operatorname{span}\left\{e_{m, 2 m+n+j}+e_{2 m+j, 2 m}, e_{m, 2 m+j}-e_{2 m+n+j, 2 m}, j=1, \ldots, n,\right. \\
& \left.e_{k, 2 m}-e_{m, m+k}, e_{m, k}-e_{m+k, 2 m}, k=1, \ldots, m-1,\right\},
\end{aligned}
$$

with $N=2(m+n-1)$. This case and all cases from Step 7 are isomorphic.

Step 9. Delete the nonadjacent nodes $i, j, i<j-1, j=3, \ldots, n$ from the extended distinguished Dynkin diagram. The remaining diagram is that of $\tilde{G}_{0}=C_{i} \oplus \operatorname{sl}(j-i) \oplus$ $D(m \mid n-j)$ (for $i=1$ instead of $C_{i}$ we have $A_{1}$ ). In each case there are seven $\tilde{G}_{0}$-modules $g_{k}$, one of which is invariant under $\omega$ (say $g_{1}$ ). Then one has to put $G_{0}=H+\tilde{G}_{0}+g_{1}$, and in each case one finds $G_{0} \equiv H+s l(j-i) \oplus D(m \mid n-j+i)$. Now there are four $G_{0}$-modules and $D(m \mid n)=G_{-2} \oplus G_{-1} \oplus G_{0} \oplus G_{+1} \oplus G_{+2}$. All these cases are isomorphic to those of Step 1 with $i=2, \ldots, n-1$. 
Step 10. Delete the nonadjacent nodes $i, j, i=1, \ldots, n, j=n+1, \ldots, n+m-2[$ but $(i, j) \neq(n, n+1)]$ from the extended distinguished Dynkin diagram. The remaining diagram is that of $\tilde{G}_{0}=C_{i} \oplus \operatorname{sl}(j-n \mid n-i) \oplus D_{m+n-j}$ (for $j=n+m-2$ instead of $D_{n+m-j}$ we have $\left.A_{1} \oplus A_{1}\right)$. In each case there are seven $\tilde{G}_{0}$-modules $g_{k}$, one of which is invariant under $\omega$ (say $g_{1}$ ). Then one has to put $G_{0}=H+\tilde{G}_{0}+g_{1}$, and in each case one finds $G_{0} \equiv H+s l(j-n \mid n-i) \oplus D(m+n-j \mid i)$. Now there are four $G_{0}$-modules and $D(m \mid n)=$ $G_{-2} \oplus G_{-1} \oplus G_{0} \oplus G_{+1} \oplus G_{+2}$ with

$$
\begin{aligned}
G_{-1}= & \operatorname{span}\left\{e_{k l}-e_{m+l, m+k}, e_{k, m+l}-e_{l, m+k}, e_{r, 2 m+n+s}+e_{2 m+s, m+r},\right. \\
& e_{m+l, 2 m+n+p}+e_{2 m+p, l}, e_{k, 2 m+q}-e_{2 m+n+q, m+k}, \\
& e_{2 m+q, 2 m+n+p}+e_{2 m+p, 2 m+n+q}, e_{2 m+p, 2 m+q}-e_{2 m+n+q, 2 m+n+p}, \\
& k=1, \ldots, j-n, l=j-n+1, \ldots, m, r=1, \ldots, m, s=1, \ldots, n, \\
& q=1, \ldots, i, p=i+1, \ldots, n\},
\end{aligned}
$$

with $N=2(j-i)(m+n-j+i)$.

Step 11. Delete the nonadjacent nodes $i, j, i=1, \ldots, n$, and $j=n+m-1$ or $n+m$ from the extended distinguished Dynkin diagram. The remaining diagram is that of $G_{0}=$ $C_{i} \oplus \operatorname{sl}(m \mid n-i)$. There are four $G_{0}$-modules and $D(m \mid n)=G_{-2} \oplus G_{-1} \oplus G_{0} \oplus G_{+1} \oplus G_{+2}$ with

$$
\begin{aligned}
G_{-1}= & \operatorname{span}\left\{e_{k, 2 m+p}-e_{2 m+n+p, m+k}, e_{k, 2 m+n+p}+e_{2 m+p, m+k},\right. \\
& e_{2 m+p, 2 m+n+q}+e_{2 m+q, 2 m+n+p}, e_{2 m+q, 2 m+p}-e_{2 m+n+p, 2 m+n+q} \\
& k=1, \ldots, m, p=1, \ldots, i, q=i+1, \ldots, n\}
\end{aligned}
$$

for $j=m+n$ and a similar expression for $j=m+n-1$. Naturally, both cases are isomorphic and $N=2 i(m+n-i)$.

Step 12. Delete the nonadjacent nodes $i, j, i=n+1, \ldots, m+n-2, j=n+m-1$ or $n+m$ from the extended distinguished Dynkin diagram. The remaining diagram is that of $G_{0}=D(i-n \mid n) \oplus s l(m+n-i)$ (if $i=n+1$ instead of $D(i-n \mid n)$ we have $C(n+1)$ ). There are four $G_{0}$-modules and $D(m \mid n)=G_{-2} \oplus G_{-1} \oplus G_{0} \oplus G_{+1} \oplus G_{+2}$ with

$$
\begin{gathered}
G_{-1}=\operatorname{span}\left\{e_{k l}-e_{m+l, m+k}, e_{m+k, l}-e_{m+l, k}, e_{m+l, 2 m+n+p}+e_{2 m+p, l} ; e_{m+l, 2 m+p}-e_{2 m+n+p, l},\right. \\
k=1, \ldots, i-n, l=i-n+1, \ldots, m, p=1, \ldots, n\}
\end{gathered}
$$

for $j=m+n$ and a similar expression for $j=m+n-1$, both having $N=2 i(m+n-i)$. The cases here and in Step 10 with one and the same $G_{0}$ and $N$ are isomorphic.

Step 13. Delete the nonadjacent nodes $i, j, i<j-1, i=n+1, \ldots, n+m-4, j=$ $n+3, \ldots, n+m-2$ from the extended distinguished Dynkin diagram. The remaining diagram is that of $\tilde{G}_{0}=D(i-n \mid n) \oplus \operatorname{sl}(j-i) \oplus D_{m+n-j}($ for $i=n+1$ instead of $D(i-n \mid n)$ we have $C(n+1)$, for $j=m+n-2$ instead of $D_{m+n-j}$ we have $\left.A_{1} \oplus A_{1}\right)$. In each case there are seven $\tilde{G}_{0}$-modules $g_{k}$, one of which is invariant under $\omega$ (say $g_{1}$ ). Then one has to put $G_{0}=H+\tilde{G}_{0}+g_{1}$, and in each case one finds $G_{0} \equiv H+s l(j-i) \oplus D(m-j+i \mid n)$. Now there are four $G_{0}$-modules and $D(m \mid n)=G_{-2} \oplus G_{-1} \oplus G_{0} \oplus G_{+1} \oplus G_{+2}$ with

$$
\begin{gathered}
G_{-1}=\operatorname{span}\left\{e_{k l}-e_{m+l, m+k}, e_{m+k, l}-e_{m+l, k}, e_{m+l, 2 m+n+q}+e_{2 m+q, l}, e_{m+l, 2 m+q}-e_{2 m+n+q, l},\right. \\
k=1, \ldots, i-n, j-n+1, \ldots, m, l=i-n+1, \ldots, j-n, q=1, \ldots, n\}
\end{gathered}
$$


with $N=2(j-i)(m+n-j+i)$. All of these are isomorphic to cases in Step 12 .

Step 14. If we delete three or more nodes from the extended distinguished Dynkin diagram, the corresponding $\mathbb{Z}$-grading of $D(m \mid n)$ has no longer the required properties (i.e. there are non-zero subspaces $G_{i}$ with $|i|>2$ ).

Step 15. Also here, we have considered all the non-distinguished Dynkin diagrams and their extensions, following [17. Repeating our procedure for all of them, leads again to a lot of case studies, most of which are isomorphic to results of the previous steps. There is, however, one extra case that is not covered in the previous steps, and that we shall briefly describe. Consider the non-distinguished Dynkin diagrams of $D(m \mid n)$ of the following form:

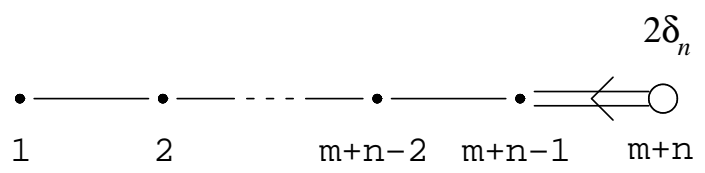

In this diagram, each dot stands for a white or gray circle, depending upon whether the corresponding simple root is even or odd. All these other simple roots are of the form $\eta-\eta^{\prime}$, where $\eta$ and $\eta^{\prime}$ can be a $\epsilon_{i}$ or a $\delta_{j}$. For example, root $m+n-1$ is either $\delta_{n-1}-\delta_{n}$ (in which case the circle is white) or $\epsilon_{m}-\delta_{n}$ (in which case it is gray). Deleting node $i(i=1, \ldots, m+n-2)$ yields the (non-distinguished) Dynkin diagram of $G_{0}=\operatorname{sl}(k \mid l) \oplus D(m-k \mid n-l)$. Most of these cases are isomorphic to those already found in Step 10 or 11. However, the case $G_{0}=H+s l(m-1 \mid l) \oplus D(1 \mid n-l)=H+\operatorname{sl}(m-1 \mid l) \oplus C(n-l+1)(l=0, \ldots, n-1)$ did not occur before. This adds a new case to the classification, for which the length of the grading is 5. From a detailed analysis, it follows that this is the only extra case that can be obtained from the non-distinguished Dynkin diagrams (extended or not).

\section{The Lie superalgebras $C(n)$}

Let $G=C(n)=D(1 \mid n-1)=\operatorname{osp}(2 \mid 2 n-2)$. For a description of the root vectors, we refer to the previous section. The even roots are of the form $\pm \delta_{j} \pm \delta_{k}(j, k=1, \ldots, n-1)$ and the odd roots are $\pm \epsilon \pm \delta_{k}(k=1, \ldots, n-1)$. This Lie superalgebra is treated separately from $D(m \mid n)$ because its distinguished Dynkin diagram is different (see Table 1), and its structure is also different (it is a type I Lie superalgebra [4]).

Step 1. Delete node 1 from the distinguished Dynkin diagram. Then the corresponding diagram is that of $G_{0}=C_{n-1}$. There are only two $G_{0}$-modules and $C(n)$ has the grading $C(n)=G_{-1} \oplus G_{0} \oplus G_{+1}$, where

$$
\begin{aligned}
& G_{-1}=\operatorname{span}\left\{e_{1, i+2}-e_{n+1+i, 2}, e_{1, n+1+i}+e_{i+2,2}, i=1, \ldots, n-1\right\}, \\
& G_{+1}=\operatorname{span}\left\{e_{2, n+1+i}+e_{i+2,1}, e_{2, i+2}-e_{n+1+i, 1}, i=1, \ldots, n-1\right\},
\end{aligned}
$$

with $N=2(n-1)$. Putting

$$
\begin{array}{ll}
c_{-, i}^{-}=e_{1,2+i}-e_{n+1+i, 2}, & c_{+, i}^{-}=e_{1, n+1+i}+e_{2+i, 2}, \\
c_{-, i}^{+}=e_{2, n+1+i}+e_{2+i, 1}, & c_{+, i}^{+}=e_{2,2+i}-e_{n+1+i, 1},
\end{array}
$$

the operators $c_{\xi i}^{ \pm}, \xi= \pm, i=1, \ldots, n-1$, satisfy the following relations:

$$
\left[\left\{c_{\xi i}^{-}, c_{\eta j}^{+}\right\}, c_{\epsilon k}^{+}\right]=\xi \delta_{\xi \eta} \delta_{i j} c_{\epsilon k}^{+}-\epsilon \delta_{\xi \epsilon} \delta_{i k} c_{\eta j}^{+}+\eta \delta_{-\eta \epsilon} \delta_{j k} c_{-\xi i}^{+}
$$




$$
\begin{aligned}
& {\left[\left\{c_{\xi i}^{-}, c_{\eta j}^{+}\right\}, c_{\epsilon k}^{-}\right]=-\xi \delta_{\xi \eta} \delta_{i j} c_{\epsilon, k}^{-}+\eta \delta_{\eta \epsilon} \delta_{j k} c_{\xi i}^{-}+\epsilon \delta_{-\xi \epsilon} \delta_{i k} c_{-\eta j}^{-}} \\
& \left\{c_{-i}^{-}, c_{+j}^{+}\right\}=\left\{c_{-j}^{-}, c_{+i}^{+}\right\}, \quad\left\{c_{+i}^{-}, c_{-j}^{+}\right\}=\left\{c_{+j}^{-}, c_{-i}^{+}\right\} \\
& \left\{c_{\xi i}^{-}, c_{\eta j}^{-}\right\}=\left\{c_{\xi i}^{+}, c_{\eta j}^{+}\right\}=0 .
\end{aligned}
$$

(here and throughout, by convention, $\xi, \eta, \epsilon$ are written as \pm when used as subscripts, and as \pm 1 when used algebraically as multipliers).

Step 2. Delete node $i, i=2, \ldots, n-1$, from the distinguished Dynkin diagram. The corresponding diagram is the Dynkin diagram of $G_{0}=H+\operatorname{sl}(1 \mid i-1) \oplus C_{n-i}($ if $i=n-1$, $\left.C_{n-i}=A_{1}\right)$. There are four simple $G_{0}$-modules and $C(n)=G_{-2} \oplus G_{-1} \oplus G_{0} \oplus G_{+1} \oplus G_{+2}$ where

$$
\begin{aligned}
G_{-1}= & \operatorname{span}\left\{e_{j+2, k+2}-e_{n+1+k, n+1+j}, e_{j+2, n+1+k}+e_{k+2, n+1+j}, e_{1, k+2}-e_{n+1+k, 2},\right. \\
& \left.e_{1, n+1+k}+e_{k+2,2}, ; j=1, \ldots, i-1, k=i, \ldots, n-1\right\}
\end{aligned}
$$

with $N=2 i(n-i)$. An interesting case is that with $i=n-1$ and $N=2(n-1)$.

Step 3. Delete node $n$ from the distinguished Dynkin diagram. Then the corresponding diagram is that of $G_{0}=s l(1 \mid n-1)$. There are only two $G_{0}$-modules and $C(n)$ has grading $C(n)=G_{-1} \oplus G_{0} \oplus G_{+1}$, where

$$
\begin{aligned}
G_{-1}= & \operatorname{span}\left\{e_{k+2, n+1+l}+e_{l+2, n+1+k}, k \leq l=1, \ldots, n-1,\right. \\
& \left.e_{1, n+1+j}+e_{j+2,2}, j=1, \ldots, n-1\right\} .
\end{aligned}
$$

This is also an interesting case, since there are $N=\frac{n(n+1)}{2}-1$ supercommuting annihilation (resp. creation) operators.

Step 4. Upon deleting two nodes $i$ and $j$ (except $i=1, j=n$ ) or more from the distinguished Dynkin diagram of $C(n)$, the corresponding $\mathbb{Z}$-gradings have no longer the required property (there are non-zero $G_{i}$ with $|i|>2$ ).

Step 5. Delete node 1 and $n$ from the distinguished Dynkin diagram. We have $G_{0}=$ $H+\operatorname{sl}(n-1)$. There are six simple $G_{0}$-modules. All the possible combinations of these modules give rise to gradings of the form $C(n)=G_{-2} \oplus G_{-1} \oplus G_{0} \oplus G_{+1} \oplus G_{+2}$. There are essentially three different ways in which these $G_{0}$-modules can be combined. To characterize these three cases, it is sufficient to give only $G_{-1}$ :

$$
\begin{aligned}
G_{-1}= & \operatorname{span}\left\{e_{j+2, n+k+1}+e_{k+2, n+j+1}, j \leq k=1, \ldots, n-1,\right. \\
& \left.e_{1, i+2}-e_{n+i+1,2}, i=1, \ldots, n-1\right\},
\end{aligned}
$$

with $N=n(n+1) / 2-1$;

$$
G_{-1}=\operatorname{span}\left\{e_{1, k+2}-e_{n+k+1,2}, e_{2, k+2}-e_{n+k+1,1}, k=1, \ldots, n-1\right\},
$$

with $N=2(n-1)$;

$$
\begin{aligned}
G_{-1}= & \operatorname{span}\left\{e_{1, n+k+1}+e_{k+2,2}, \quad k=1, \ldots, n-1,\right. \\
& \left.e_{n+l+1, p+2}+e_{n+p+1, l+2}, \quad l \leq p=1, \ldots, n-1\right\},
\end{aligned}
$$


with $N=n(n+1) / 2-1$. It is interesting to give the algebraic relations for (7.6) , since the number of creation and annihilation operators is $N=2(n-1)$. One can label the CAOs as follows $(k=1, \ldots, n-1)$ :

$$
\begin{array}{ll}
c_{-k}^{-}=e_{1, k+2}-e_{n+k+1,2}, & c_{+k}^{-}=e_{2, k+2}-e_{n+k+1,1}, \\
c_{-k}^{+}=e_{2, n+k+1}+e_{k+2,1}, & c_{+k}^{+}=e_{1, n+k+1}+e_{k+2,2} .
\end{array}
$$

The CAOs $c_{\xi k}^{ \pm}, \xi= \pm, k=1, \ldots, n-1$, satisfy the relations $(\xi, \eta, \epsilon= \pm$ or $\pm 1 ; j, k, l=$ $1, \ldots, n-1)$ :

$$
\begin{aligned}
& \left\{c_{\xi j}^{\eta}, c_{\xi k}^{\eta}\right\}=\left\{c_{-j}^{-}, c_{+k}^{+}\right\}=\left\{c_{+j}^{-}, c_{-k}^{+}\right\}=0, \\
& \left\{c_{+j}^{-}, c_{+k}^{+}\right\}=\left\{c_{-j}^{-}, c_{-k}^{+}\right\}, \quad j \neq k, \\
& \left\{c_{\xi j}^{\xi}, c_{-\xi k}^{\xi}\right\}=\left\{c_{\xi k}^{\xi}, c_{-\xi j}^{\xi}\right\}, \\
& {\left[\left\{c_{\xi j}^{\gamma}, c_{\eta k}^{\gamma}\right\}, c_{\epsilon l}^{\gamma}\right]=0,} \\
& {\left[\left\{c_{\xi j}^{\xi}, c_{-\xi k}^{\xi}\right\}, c_{\epsilon l}^{-\xi}\right]=-\xi \delta_{k l} c_{-\epsilon j}^{\xi}-\xi \delta_{j l} c_{-\epsilon k}^{\xi},} \\
& {\left[\left\{c_{\xi j}^{-}, c_{\xi k}^{+}\right\}, c_{\eta l}^{-}\right]=-\delta_{k l} c_{\eta j}^{-}-(-1)^{\delta_{\xi \eta}} \delta_{j k} c_{\eta l}^{-},} \\
& {\left[\left\{c_{\xi j}^{-}, c_{\xi k}^{+}\right\}, c_{\eta l}^{+}\right]=(-1)^{\delta_{\xi \eta}} \delta_{j k} c_{\eta l}^{+}+\delta_{j l} c_{\eta k}^{+} .}
\end{aligned}
$$

Step 6. Delete node $i, i=0,1, \ldots, n$, from the extended distinguished Dynkin diagram. The remaining diagram is that of $G_{0}=C(n), C(n), s l(2 \mid 1) \oplus C_{n-2}, C(3) \oplus C_{n-3}, \ldots, C_{n-1} \oplus A_{1}$, $C(n)$. In all these cases there is only one $G_{0}$-module, so there are no contributions to the classification.

Step 7. Delete the adjacent nodes $i$ and $i+1, i=2,3, \ldots, n-2$, from the extended distinguished Dynkin diagram. The remaining diagram is that of $\tilde{G}_{0}=H+\operatorname{sl}(2 \mid 1) \oplus C_{n-3}$ for $i=2$ and of $\tilde{G}_{0}=H+C(i) \oplus C_{n-i-1}$ for $i=3, \ldots, n-2$. In each case there are seven $\tilde{G}_{0}$-modules $g_{k}$, one of which is invariant under $\omega$ (say $g_{1}$ ). Then one has to put $G_{0}=H+\tilde{G}_{0}+g_{1}$, and in each case one finds $G_{0} \equiv H+C(n-1)$. Now there are four $G_{0}$-modules and $C(n)=G_{-2} \oplus G_{-1} \oplus G_{0} \oplus G_{+1} \oplus G_{+2}$ with

$$
\begin{aligned}
G_{-1}= & \operatorname{span}\left\{e_{i+2, n+k+1}+e_{k+2, n+i+1}, e_{i+2, k+2}-e_{n+k+1, n+i+1}, e_{1, n+i+1}+e_{i+2,2},\right. \\
& \left.e_{2, n+i+1}+e_{i+2,1} ; k \neq i=1, \ldots, n-1\right\}
\end{aligned}
$$

and $N=2(n-1)$. All these cases are mutually isomorphic.

Step 8. Delete the adjacent nodes $n-1$ and $n$ from the extended distinguished Dynkin diagram. The remaining diagram is that of $G_{0} \equiv H+C(n-1)$. This case turns out to be isomorphic to those of Step 7.

Step 9. Delete the nonadjacent nodes $i, i=2,3, \ldots, n-2$, and $j=n$ from the extended distinguished Dynkin diagram. The remaining diagram is that of $G_{0}=H+C(i) \oplus \operatorname{sl}(n-i)$ (for $i=2$ instead of $C(i)$ we have $s l(2 \mid 1)$ ). In each case there are four $G_{0}$-modules and $C(n)=G_{-2} \oplus G_{-1} \oplus G_{0} \oplus G_{+1} \oplus G_{+2}$ with

$$
\begin{aligned}
G_{-1}= & \operatorname{span}\left\{e_{k+2, l+2}-e_{n+1+l, n+1+k}, e_{n+1+k, l+2}+e_{n+l+1, k+2}, e_{1, l+2}-e_{n+l+1,2},\right. \\
& \left.e_{2, l+2}-e_{n+l+1,1} ; l=i, \ldots, n-1, k=1, \ldots, i-1\right\}
\end{aligned}
$$

and $N=2 i(n-i)$. These are all new cases. 
Step 10. Delete the nonadjacent nodes $i<j-1, i=2,3, \ldots, n-2, j=4, \ldots, n-1$ from the extended distinguished Dynkin diagram. The remaining diagram is that of $\tilde{G}_{0}=$ $H+C(i) \oplus \operatorname{sl}(j-i) \oplus C_{n-j}$ (for $i=2$ instead of $C(i)$ we have $s l(2 \mid 1)$, for $j=n-1$ instead of $C_{n-j}$ we have $\left.A_{1}\right)$. In each case there are seven $\tilde{G}_{0}$-modules $g_{k}$, one of which is invariant under $\omega$ (say $g_{1}$ ). Then one has to put $G_{0}=H+\tilde{G}_{0}+g_{1}$, and in each case one finds $G_{0} \equiv H+\operatorname{sl}(j-i) \oplus C(n-j+i)$. Now there are four $G_{0}$-modules and $C(n)=$ $G_{-2} \oplus G_{-1} \oplus G_{0} \oplus G_{+1} \oplus G_{+2}$ with

$$
\begin{aligned}
G_{-1}= & \operatorname{span}\left\{e_{k+2, l+2}-e_{n+1+l, n+1+k}, e_{n+1+k, l+2}+e_{n+l+1, k+2}, e_{1, l+2}-e_{n+l+1,2},\right. \\
& \left.e_{2, l+2}-e_{n+l+1,1} ; l=i, \ldots, j-1, k=1, \ldots, i-1, j, \ldots, n-1\right\}
\end{aligned}
$$

and $N=2(j-i)(n-j+i)$. All these cases are amongst those of Step 10 .

Step 11. Upon deleting three or more nodes from the extended distinguished Dynkin diagram of $C(n)$, the corresponding $\mathbb{Z}$-gradings have no longer the required property (there are non-zero $G_{i}$ with $|i|>2$ ).

The other non-distinguished choices for the simple root systems give no new results.

\section{Summary and conclusions}

Our analysis has led to a complete classification of all GQS associated with the basic classical Lie superalgebras. Some cases in our classification have appeared earlier as examples, e.g. para-Bose statistics (5.1), A-superstatistics (3.4) in [11, 15], and the combined paraBose/para-Fermi case (4.7) in [19]. Some other examples are also rather simple, e.g. the alternatives to $A$-superstatistics in (3.5), a statistics with two kind of particles in (3.6), and the statistics related to $C(n)$ superalgebras (7.2) and (7.8).

Although the detailed analysis in the previous sections was necessary to present the complete solution, it is convenient to summarize the final results in a table. Table 2 recapitulates the classification of all GQS. From this table, it follows that many of the earlier cases can be somewhat unified in a simple form, provided one makes use of the common isomorphisms for Lie algebras and Lie superalgebras (such as $D_{1}=C_{1}=A_{1}, D_{2}=A_{1} \oplus A_{1}, B(m \mid 0)=B_{m}$, $D(1 \mid 1)=C(2)=s l(1 \mid 2)$ etc.). For $A(m \mid n)$, there are essentially two distinct cases. Either $G_{0}=H+s l(k \mid l) \oplus s l(p \mid q)$, in which case the grading has length 3 and $G_{ \pm 1}$ is fixed by $G_{0}$. Or else $G_{0}=H+s l(k \mid l) \oplus s l(p \mid q) \oplus s l(r \mid s)$, in which case the grading has length 5 . In this second case, there are always three ways of combining the $G_{0}$-modules in order to give some $G_{-1}$. For $B(m \mid n)$, all the cases are characterized by a $G_{0}$ of the form $G_{0}=H+s l(k \mid l) \oplus B(m-k, n-l)$. This includes cases such as $s l(l) \oplus B(m \mid n-l), s l(k \mid n) \oplus B_{m-k}$ (Step 1), $B(m \mid n-1)$ (Step 4) and $B(m-1 \mid n)$ (Step 5). Also the results for the remaining Lie superalgebras can be neatly summarized. Note that for $D(m \mid n)$ (and for $C(n)=D(1 \mid n-1)$ ) there is one $G_{0}$ which gives rise not only to different possibilities for $G_{-1}$ but even for $N$.

A striking property, see Table 2, is that all basic classical Lie superalgebras, except $B(0 \mid n)$, allow a GQS with a grading of length 3; in other words, a GQS with supercommuting creation and annihilation operators.

Note that a set of CAOs together with a complete set of relations $\mathcal{R}$ unambiguously describes the Lie superalgebra. So each case of our classification also gives the description of a Lie superalgebra in terms of a number of generators subject to certain relations. This can also be reformulated in terms of the notion of Lie supertriple systems [20]. In fact, in our 
case the subspace $G_{-1} \oplus G_{+1}$ (i.e. the subspace spanned by all CAOs) is a Lie supertriple system for the universal enveloping algebra $U(G)$.

Just as in [2, we have dealt only with a mathematical definition of generalized quantum statistics. In order to talk about a quantum statistics in the physical sense, one should take into account additional requirements for the CAOs, related to certain quantization postulates [8]. These conditions are related to the existence of state spaces (Fock spaces), in which the CAOs act in such a way that the corresponding observables are Hermitian operators. We refer to section VII of [2] for a discussion on this. We hope that some cases of our classification will yield interesting GQS also from this point of view.

As a second application, we mention the possible solutions of Wigner Quantum Systems [21]. Roughly speaking, the compatibility conditions (CCs) to be satisfied by a Wigner Quantum Oscillator system (see formula (3.7) in [22]) are written in terms of certain odd operators $A_{i}^{ \pm}$; furthermore, these CCs are special triple relations. So it is of importance to investigate which triple relations $\mathcal{R}$ of our current classification of GQS could provide special solutions of these CCs. It is known, for instance, that there is a $s l(1 \mid n)$ solution [21]-[23] or a $s l(n \mid 3)$ solution [24]. Obviously, for a possible candidate solution all the CAOs of $\mathcal{R}$ should be odd operators. Let us briefly describe the GQSs of the current classification which have only odd CAOs. Then $G_{-1}$ and $G_{+1}$ are odd subspaces, and by the grading condition it follows automatically that the GQS grading (2.3) is consistent with the $\mathbb{Z}_{2}$-grading. So our problem reduces to selecting those GQS from our classification with a consistent $\mathbb{Z}$-grading. This is not too difficult.

For $A(m \mid n)$, one can consider $i=m+1$ in Step 1. Then all elements of $G_{-1}$ in (3.2) are odd; $G_{0}=H+s l(m+1) \oplus \operatorname{sl}(n+1)$, and the grading has length 3 (the case $m=0$ corresponds to [11], 21]-23]). Alternatively, one can consider the cases $(i, j)=(i, m+1)$ or $(i, j)=(m+1, j)(i<j)$ of Step 2. In the case $(i, j)=(i, m+1)$, the elements of $G_{-1}$ given by (3.9) are all odd, and $G_{0}=H+\operatorname{sl}(i) \oplus \operatorname{sl}(m+1-i) \oplus \operatorname{sl}(n+1)$. In the case $(i, j)=(m+1, j)$, the elements of $G_{-1}$ given by (3.8) are all odd, and $G_{0}=H+\operatorname{sl}(m+1) \oplus \operatorname{sl}(k) \oplus \operatorname{sl}(n+1-k)$ with $k=j-m-1$. For $B(m \mid n)$, the case $i=n$ in Step 1 leads to a $G_{-1}$ with only odd elements in (4.2). Note that in this case $G_{0}=H+s l(n) \oplus B_{m}$. For $B(0 \mid n)$, this corresponds to taking $i=n$ in Step 1, so that $G_{-1}$ in (5.1) has odd elements only and $G_{0}=H+\operatorname{sl}(n)$ (this is the para-Bose case). For $D(m \mid n)$, the case $i=n$ in Step 1 leads to a $G_{-1}$ in (6.1) with odd elements only, and with $G_{0}=H+s l(n) \oplus D_{m}$. There is a second solution here, namely the case $i=n$ in Step 11; then $G_{-1}$, given by (6.11), has odd elements only, and $G_{0}=H+\operatorname{sl}(m) \oplus C_{n}$. Finally, for $C(n)$ the solution provided in Step 1 has only odd elements for $G_{-1}$, see (17.1), with $G_{0}=H+C_{n-1}$. Also here there is a second solution, given in Step 5 with $G_{-1}$ of the form (7.6) and $G_{0}=H+\operatorname{sl}(n-1)$. To conclude, all the basic classical Lie superalgebras have GQSs with odd CAOs only. Whether all these cases provide special solutions to the CCs of the Wigner Quantum Oscillator will be treated elsewhere.

\section{Acknowledgments}

N.I. Stoilova was supported by a project from the Fund for Scientific Research - Flanders (Belgium). 


\section{References}

[1] H.S. Green, Phys. Rev. 90, 270 (1953).

[2] N.I. Stoilova and J. Van der Jeugt, J. Math. Phys. 46, 033501 (2005).

[3] S. Kamefuchi and Y. Takahashi, Nucl. Phys. 36, 177 (1962); C. Ryan and E.C.G. Sudarshan, Nucl. Phys. 47, 207 (1963).

[4] V.G. Kac, Adv. Math. 26, 8 (1977).

[5] A.Ch. Ganchev and T.D. Palev, J. Math. Phys. 21, 797 (1980).

[6] Y. Ohnuki, S. Kamefuchi, Quantum Field Theory and parastatistics, Springer, Berlin (1982).

[7] T.D. Palev, Lie algebraical aspects of the quantum statistics, (Habilitation thesis, Inst. Nucl. Research and Nucl. Energy, Sofia, 1976, in Bulgarian).

[8] T.D. Palev, Lie algebraic aspects of quantum statistics. Unitary quantization (Aquantization), Preprint JINR E17-10550 (1977) and hep-th/9705032.

[9] T.D. Palev, Czech. J. Phys. B 29, 91 (1979).

[10] T.D. Palev, Rep. Math. Phys. 18, 117 (1980); 18, 129 (1980).

[11] T.D. Palev, J. Math. Phys. 21, 1293 (1980).

[12] T.D. Palev, J. Van der Jeugt, J. Math. Phys. 43, 3850 (2002).

[13] A. Jellal, T.D. Palev and J. Van der Jeugt, J. Phys. A: Math. Gen. 34, 10179 (2001); preprint hep-th/0110276.

[14] T.D. Palev, N.I. Stoilova and J. Van der Jeugt, J. Phys. A: Math. Gen. 33, 2545 (2000).

[15] T.D. Palev, N.I. Stoilova and J. Van der Jeugt, J. Phys. A: Math. Gen. 36, 7093 (2003).

[16] N. Jacobson, Am. J. Math. 71, 149 (1949).

[17] D.A. Leites, M.V. Saveliev and V.V. Serganova, Serpuhov preprint 85-81 (1985); J.W. Van de Leur, Contragredient Lie superalgebras of finite growth, Utrecht thesis (1985);

L. Frappat, A. Sciarrino and P. Sorba, Commun. Math. Phys. 121, 457 (1989).

[18] J. Van der Jeugt, J. Math. Phys. 28, 292 (1987).

[19] T.D. Palev, J. Math. Phys. 23, 1100 (1982).

[20] S. Okubo, J. Math. Phys. 35, 2785 (1994).

[21] T.D. Palev, J. Math. Phys. 23, 1778 (1982).

[22] T.D. Palev and N.I. Stoilova, J. Math. Phys. 38, 2506 (1994). 
[23] R.C. King, T.D. Palev, N.I. Stoilova and J. Van der Jeugt, J. Phys. A: Math. Gen. 36, 4337 (2003); 36, 11999 (2003).

[24] T.D. Palev and N.I. Stoilova, Rep. Math. Phys. 49, 395 (2002). 
Table 1. Classical Lie superalgebras, their (extended) Dynkin diagrams with a labeling of the nodes and the corresponding simple roots.

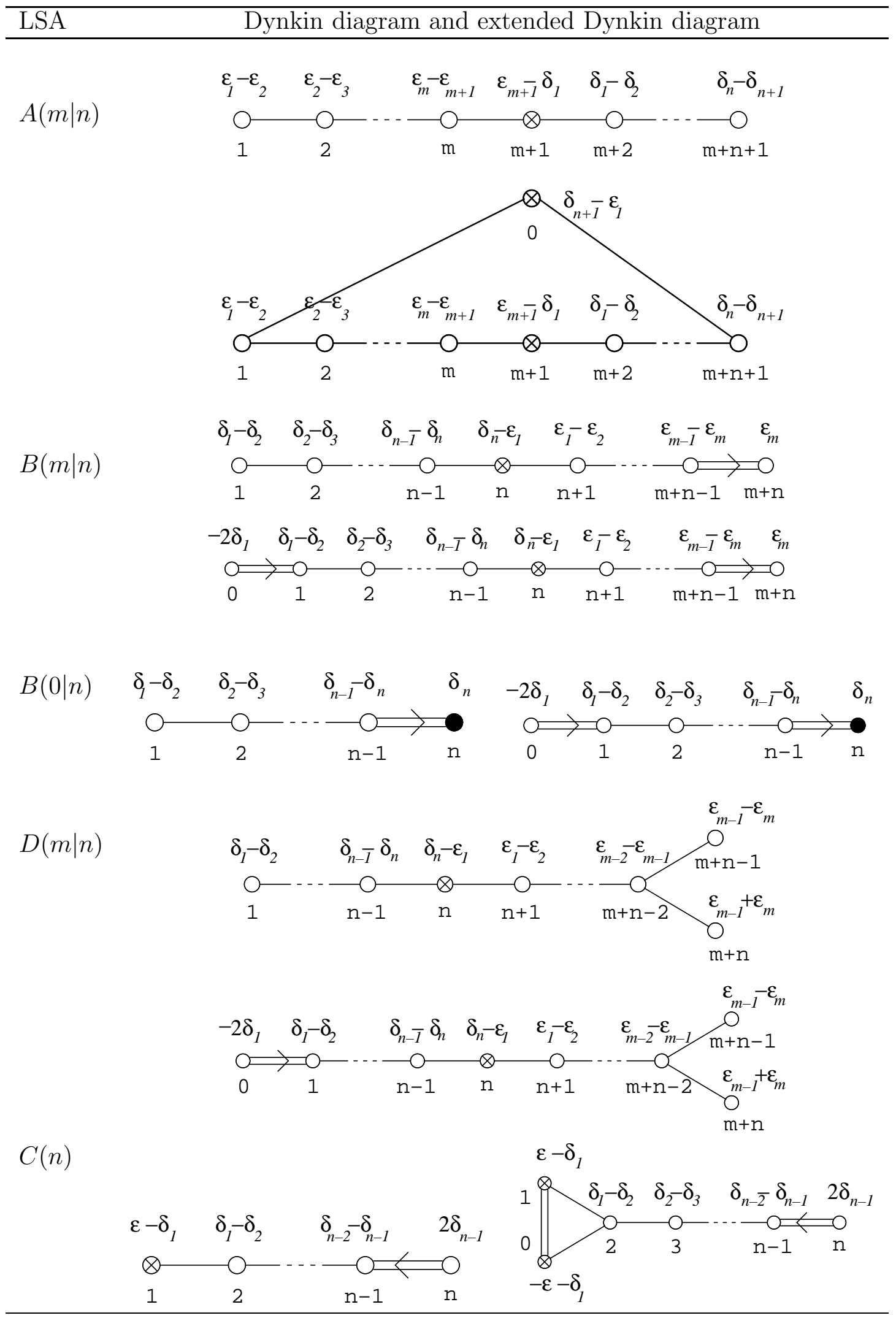


Table 2. Summary of the classification: all non-isomorphic GQS associated with a classical Lie superalgebra (LSA) are given. For each GQS, we list: the subalgebra $G_{0}$ (each $G_{0}$ contains the complete Cartan subalgebra $H$, so we only list the remaining part of $G_{0}=$ $H+\cdots)$; the length $\ell$ of the $Z$-grading; the number $N$ of annihilation operators; the reference in the text where (an example of) $G_{-1}$ can be found.

\begin{tabular}{|c|c|c|c|c|}
\hline LSA & $G_{0}=H+\cdots$ & $\ell$ & $N$ & $G_{-1}$ \\
\hline \multirow[t]{4}{*}{ 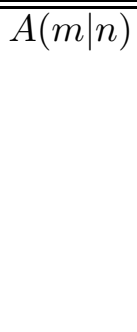 } & $\begin{array}{l}s l(k \mid l) \oplus \operatorname{sl}(p \mid q) \\
(k+p=m+1, l+q=n+1 \\
k+l \neq 0, p+q \neq 0)\end{array}$ & 3 & $(k+l)(p+q)$ & (3.2) or $(3.12)$ \\
\hline & $\operatorname{sl}(k \mid l) \oplus \operatorname{sl}(p \mid q) \oplus \operatorname{sl}(r \mid s)$ & 5 & $(k+l)(p+q+r+s)$ & (3.7), (3.13) \\
\hline & $(k+p+r=m+1$ & 5 & $(p+q)(k+l+r+s)$ & (3.8), (3.14) \\
\hline & $\begin{array}{l}l+q+s=n+1 \\
k+l \neq 0, p+q \neq 0, r+s \neq 0)\end{array}$ & 5 & $(r+s)(k+l+p+q)$ & (3.9), (3.15) \\
\hline \multirow[t]{2}{*}{$B(m \mid n)$} & $\begin{array}{l}s l(k \mid l) \oplus B(m-k \mid n-l) \\
(k=0, \ldots, m ; l=0, \ldots, n \\
(k, l) \notin\{(0,0),(1,0)\})\end{array}$ & 5 & $(k+l)(2 m-2 k+2 n-2 l+1)$ & $\begin{array}{l}\text { (4.2), (4.3), } \\
\text { (4.11), (4.12) }\end{array}$ \\
\hline & $B(m-1 \mid n) \quad[(k, l)=(1,0)]$ & 3 & $2 m+2 n-1$ & (4.10) \\
\hline$B(0 \mid n)$ & $\begin{array}{l}s l(i) \oplus B(0 \mid n-i) \\
(i=1, \ldots, n)\end{array}$ & 5 & $i(2 n-2 i+1)$ & (5.1) \\
\hline \multirow[t]{5}{*}{$\overline{D(m \mid n)}$} & $\begin{array}{l}s l(k \mid l) \oplus D(m-k \mid n-l) \\
(k=0,1, \ldots, m ; \\
l=0,1, \ldots, n \\
(k, l) \notin\{(0,0),(1,0),(m-1, n),(m, n)\})\end{array}$ & 5 & $2(k+l)(m+n-k-l)$ & $\begin{array}{l}(6.10),(6.11) \\
(6.1),(6.2) \\
(6.12)\end{array}$ \\
\hline & $D(m-1 \mid n) \quad[(k, l)=(1,0)]$ & 3 & $2(m+n-1)$ & $(6.8),(6.9)$ \\
\hline & {$[(k, l)=[m, n)]$} & 3 & $(m+n)(m+n+1) / 2-m$ & $(6.3),(6.4)$ \\
\hline & $s l(m-1 \mid n) \quad[(k, l)=(m-1, n)]$ & 5 & $(m+n)(m+n+1) / 2-m$ & $(6.6),(6.7)$ \\
\hline & $s l(m-1 \mid n) \quad[(k, l)=(m-1, n)]$ & 5 & $2(m+n-1)$ & (6.5) \\
\hline \multirow[t]{5}{*}{$C(n)$} & $\begin{array}{l}s l(k \mid l) \oplus D(1-k \mid n-1-l) \\
(k=0,1 ; l=1, \ldots, n-2)\end{array}$ & 5 & $2(k+l)(n-k-l)$ & $\begin{array}{l}(7.3),(7.9) \\
(7.10),(7.11)\end{array}$ \\
\hline & $C_{n-1} \quad[(k, l)=(1,0)]$ & 3 & $2(n-1)$ & (7.1) \\
\hline & {$[(k, l)=(1, n-1)]$} & 3 & $n(n+1) / 2-1$ & (7.4) \\
\hline & $s l(n-1) \quad[(k, l)=(0, n-1)]$ & 5 & $n(n+1) / 2-1$ & $(7.5),(7.7)$ \\
\hline & {$[(k, l)=(0, n-1)]$} & 5 & $2(n-1)$ & $(7.6)$ \\
\hline
\end{tabular}

